\title{
IDENTIFICATION OF HAMMERSTEIN SYSTEMS WITH QUANTIZED OBSERVATIONS*
}

\author{
YANLONG ZHAO ${ }^{\dagger}$, JI-FENG ZHANG ${ }^{\dagger}$, LE YI WANG ${ }^{\ddagger}$, AND G. GEORGE YIN $§$
}

\begin{abstract}
This work is concerned with identification of Hammerstein systems whose outputs are measured by quantized sensors. The system consists of a memoryless nonlinearity that is polynomial and possibly noninvertible, followed by a linear subsystem. The parameters of linear and nonlinear parts are unknown but have known orders. Input design, identification algorithms, and their essential properties are presented under the assumptions that the distribution function of the noise is known and the quantization thresholds are known. The concept of strongly scaled full rank signals is introduced to capture the essential conditions under which the Hammerstein system can be identified with set-valued observations. Under strongly scaled full rank conditions, a strongly convergent algorithm is constructed. Asymptotic consistency and efficiency of the algorithm are investigated.
\end{abstract}

Key words. identification, quantized observations, Hammerstein systems, parameter estimation, quantization thresholds, strongly scaled full rank signals

AMS subject classifications. 93E12, 93C55, 62L20

DOI. $10.1137 / 070707877$

1. Introduction. Quantized sensors are commonly employed in practical systems since they are more cost effective than regular sensors. In some applications, they are the only type of sensors available during real-time operations [24]. More importantly, quantization is a fundamental building block for communication channels. Consequently, understanding system identification under quantized observations is essential for studying identification of systems involving communication channels.

Quantized observations supply very limited information on the system outputs, and hence introduce difficulties in system identification. Classical system identification methods, such as least-squares algorithms, maximum likelihood methods, etc., assume that the output is measured by a linear sensor, which introduces accurate values of the system output, and construct estimation algorithms by calculating the system outputs. However, the information from quantized observations contains only a finite number of possible values instead of the accurate values of the system outputs, making it necessary to develop new methodologies and algorithms, and to ensure convergence of estimates.

\footnotetext{
*Received by the editors November 12, 2007; accepted for publication (in revised form) April 2, 2010; published electronically June 24, 2010.

http://www.siam.org/journals/sicon/48-7/70787.html

${ }^{\dagger}$ Key Laboratory of Systems and Control, Institute of Systems Science, Academy of Mathematics and Systems Science, Chinese Academy of Sciences, Beijing 100190, China (ylzhao@amss.ac.cn, jif@ iss.ac.cn). The research of the first author was supported in part by the National Natural Science Foundation of China under 6657100 and in part by the Knowledge Innovation Program of the Chinese Academy of Sciences. The research of the second author was supported in part by the National Natural Science Foundation of China (under 60821091 and 60934006) and in part by the National Laboratory of Space Intelligent Control.

${ }_{\ddagger}^{\ddagger}$ Department of Electrical and Computer Engineering, Wayne State University, Detroit, MI 48202 (lywang@ece.eng.wayne.edu). The research of this author was supported in part by the National Science Foundation under ECS-0329597, DMS-0624849, in part by the Michigan Economic Development Council, and in part by the Air Force Office of Scientific Research under FA9550-10-1-0210.

$\S$ Department of Mathematics, Wayne State University, Detroit, MI 48202 (gyin@math.wayne. edu). The research of this author was supported in part by the National Science Foundation under DMS-0907753, and in part by the Air Force Office of Scientific Research under FA9550-10-1-0210.
} 
The first comprehensive treatment on identification with binary-valued observations was presented in [24]. The identification problem with binary-valued observations was studied under bounded and stochastic noises, which introduced deterministic and stochastic frameworks, respectively. The identification errors, time complexity, input design, and impact of disturbances and unmodeled dynamics on identification accuracy and complexity for linear systems that are modeled by impulse responses were investigated. Along the line of a stochastic framework, the work was extended to rational models and unknown noise distributions in [22]. Recently, the methodologies have been extended to system identification with quantized observations in [21]. Most significantly, the optimality of the identification algorithms has been established by showing that the Cramér-Rao lower bound is asymptotically achieved [21], and the relationship between the space complexity and time complexity is clarified in [23]. The work on nonlinear systems with binary-valued observations started with Wiener systems in [26]. Compared to [24], the main difficulty of Wiener system identification is how to deal with the nonlinearity. The idea of scaled full rank signals was employed to overcome this difficulty. It was shown that under scaled full rank signals, identification of unknown parameters can be transformed, in an invertible mapping, into a number of simplified core identification problems involving certain intermediate variables, which are solved with the methods of [24].

This paper studies identification of Hammerstein systems whose outputs are measured by quantized sensors. Hammerstein systems consist of a static nonlinear block followed by a linear dynamic system. They typically represent, but are certainly not limited to, linear systems with memoryless nonlinear actuators, which were first carried out in [10]. Consequently, this paper deals with identification of systems with both nonlinear actuators and nonlinear sensors. In other words, we are in fact dealing with Hammerstein-Wiener systems. When the output of a Hammerstein system must be measured by a quantized sensor or sent through a communication channel, it can be represented as a Hammerstein system with quantized observations. Consequently, understanding identification of Hammerstein systems with quantized observations will be essential for studying both identification of nonlinear systems and impact of communication channels on system models.

Identification of Hammerstein systems, together with Wiener systems, has been studied extensively. References $[9,16,17]$ contain some fundamental results on identification of such systems, covering a wide range of identification entities, different nonlinear functions, and efficient properties. Identification methodologies used for Hammerstein and Wiener structures may be loosely classified by iterative algorithms [8, 12], correlation techniques [4], stochastic recursive algorithms [5, 7, 13], least-squares estimation and singular value decomposition methods $[1,11,14]$, and frequency-domain identification methods $[1,19]$, etc. All of these approaches require output measurements by linear sensors. This paper considers quantized output observations. Although such output nonlinearity may be viewed generically as a Hammerstein-Wiener structure, existing literature on identification of HammersteinWiener systems require the output nonlinearities to be smooth [1, 2]. However, a switching nonlinearity, whose output takes only a finite number of values, is fundamentally different from smooth nonlinearities that can provide much more information, and requires new methodologies in developing input design and identification algorithms. Moreover, this work differs from identification of Wiener systems by more involved input design. In essence, a periodic full rank input may lose its rank after passing through the input nonlinearity, rendering inapplicable the main ideas of [26] for identifying Wiener systems. 
This paper models the input nonlinearity by polynomial and possibly noninvertible nonlinearities. This model can be considered as an approximation of other more general nonlinear functions and has the advantages of representing function parameters in a linear structure. As such, it can simplify identification algorithm development and has been adopted extensively in studies of Hammerstein systems. Under this polynomial model of nonlinear functions, the concept of strongly scaled full rank inputs is introduced, under which the system parameters become identifiable with quantized observations. It is shown that the parameters of the linear part can be estimated first. The nonlinear polynomial function can then be identified afterwards based on the estimates of the linear part parameters.

The rest of the paper is organized as follows. The structure of Hammerstein models using quantized observations is described in section 2. The concepts of strongly full rank signals and their essential properties are introduced in section 3. Under strongly full rank inputs, estimation algorithms of unknown parameters based on individual thresholds are constructed in section 4 . Estimation errors of these algorithms are established. The estimates are integrated in an algorithm called optimal quasiconvex combination in section 5. The resulting estimates are shown to be strongly convergent (in the sense of convergence with probability one). Their efficiency is also investigated. The algorithms are expanded in section 6 to derive identification algorithms for both the linear and nonlinear parts. Illustrative examples are presented in section 7 on the input design and convergence properties of the methodologies and algorithms developed in this paper. Finally, section 8 provides a brief summary of the findings of this paper.

2. Problem formulation. Consider the single-input-single-output system in Figure 1, in which the linear dynamics is a finite impulse response system and input nonlinearity is a polynomial model

$$
\left\{\begin{array}{l}
y(k)=\sum_{i=0}^{n-1} a_{i} x(k-i)+d(k), \\
x(k)=b_{0}+\sum_{j=1}^{m} b_{j} u^{j}(k), \quad b_{m}=1,
\end{array}\right.
$$

where $u(k)$ is the input, $x(k)$ the intermediate variable, and $d(k)$ the measurement noise. Both $n$ and $m$ are known. In this paper, the superscript represents power, hence $u^{j}(k)$ is the $j$ th power $u^{j}(k)=(u(k))^{j}$. For a matrix $M, M^{T}$ denotes its transpose.

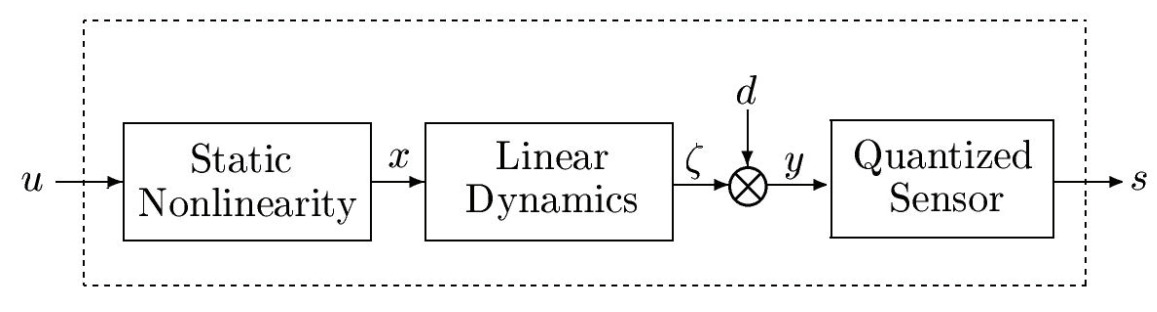

FIG. 1. Hammerstein systems with quantized observations. 
The output $y(k)$ is measured by a sensor ${ }^{1}$ with $l$ thresholds $C_{1}<\cdots<C_{l}$, which is represented by the indicator functions

$$
s_{i}(k)=\mathcal{S}\left(y(k), C_{i}\right)=I_{\left\{y(k) \leq C_{i}\right\}}, \quad i=1, \ldots, l,
$$

where

$$
I_{\{y(k) \in A\}}= \begin{cases}1 & \text { if } y(k) \in A \\ 0 & \text { otherwise. }\end{cases}
$$

Denote $\theta=\left[a_{0}, \ldots, a_{n-1}\right]^{T}, \phi_{0}(k)=[1, \ldots, 1]^{T}$, and $\phi_{j}(k)=\left[u^{j}(k), \ldots, u^{j}(k-\right.$ $n+1)]^{T}, j=1, \ldots, m$. Then

$$
\begin{aligned}
y(k) & =\sum_{i=0}^{n-1} a_{i}\left(b_{0}+\sum_{j=1}^{m} b_{j} u^{j}(k-i)\right)+d(k) \\
& =b_{0} \sum_{i=0}^{n-1} a_{i}+\sum_{j=1}^{m} b_{j} \sum_{i=0}^{n-1} a_{i} u^{j}(k-i)+d(k) \\
& =\sum_{j=0}^{m} b_{j} \phi_{j}^{T}(k) \theta+d(k) .
\end{aligned}
$$

By using the vector notation for $k=1,2, \ldots, j=0, \ldots, m$, and $i=1, \ldots, l$,

$$
\begin{aligned}
& Y(k)=[y(2(k-1)(m+1) n+n), \ldots, y(2 k(m+1) n+n-1)]^{T}, \\
& \Phi_{j}(k)=\left[\phi_{j}(2(k-1)(m+1) n+n), \ldots, \phi_{j}(2 k(m+1) n+n-1)\right]^{T}, \\
& D(k)=[d(2(k-1)(m+1) n+n), \ldots, d(2 k(m+1) n+n-1)]^{T}, \\
& S_{i}(k)=\left[s_{i}(2(k-1)(m+1) n+n), \ldots, s_{i}(2 k(m+1) n+n-1)\right]^{T}, \\
& Y(k), D(k), S_{i}(k) \in \mathbb{R}^{2 n(m+1)}, \quad \Phi_{j}(k) \in \mathbb{R}^{2 n(m+1) \times n},
\end{aligned}
$$

we can rewrite (2.3) in a block form as

$$
Y(l)=\sum_{j=0}^{m} b_{j} \Phi_{j}(l) \theta+D(l)
$$

The purpose of this paper is to develop identification algorithms for estimating the linear part parameter $\theta$ and the nonlinear part parameter $\eta=\left[b_{0}, \ldots, b_{m-1}\right]^{T}$ with the information on the input $u$ and the output $s$ of the quantized sensor.

The input signal that will be used to identify the system is a $2 n(m+1)$-periodic signal $u$ whose one-period values are $\left(v, v, \rho_{1} v, \rho_{1} v, \ldots, \rho_{m} v, \rho_{m} v\right)$, where the base vector $v=\left(v_{1}, \ldots, v_{n}\right)$ and the scaling factors $\rho_{1}, \ldots, \rho_{m}$ are to be specified. The scaling factors $1, \rho_{1}, \ldots, \rho_{m}$ are assumed to be nonzero and distinct. Under $2 n(m+1)$ periodic inputs, we have

$$
\Phi_{j}(l)=\Phi_{j}(1) \triangleq \Phi_{j}, \quad l=1,2, \ldots
$$

\footnotetext{
${ }^{1}$ The term "sensor" is used here in a generic sense. It does not have to be a physical sensor, but may be a selected partition of the output range by any method.
}

Copyright (c) by SIAM. Unauthorized reproduction of this article is prohibited. 
Thus, (2.4) can be written as

$$
Y(l)=\sum_{j=0}^{m} b_{j} \Phi_{j} \theta+D(l) \triangleq \zeta+D(l)
$$

For the above system, the identification algorithms will be divided into two steps: (i) to estimate $\zeta$ (that can be reduced to estimation of gain systems), and (ii) to estimate $\theta$ and $\eta$ from the estimated $\zeta$.

3. Strongly full rank signals. This section introduces a class of input signals, called strongly full rank signals, which will play an important role in subsequent development. First, some basic properties of periodic signals will be derived.

An $n \times n$ generalized circulant matrix (see [15])

$$
T=\left[\begin{array}{ccccc}
v_{n} & v_{n-1} & v_{n-2} & \cdots & v_{1} \\
q v_{1} & v_{n} & v_{n-1} & \ddots & v_{2} \\
q v_{2} & q v_{1} & v_{n} & \ddots & v_{3} \\
\vdots & \ddots & \ddots & \ddots & \vdots \\
q v_{n-1} & q v_{n-2} & q v_{n-3} & \cdots & v_{n}
\end{array}\right]
$$

is completely determined by its first row $\left[v_{n}, \ldots, v_{1}\right]$ and $q$, which will be denoted by $\mathbf{T}\left(q,\left[v_{n}, \ldots, v_{1}\right]\right)$. In the special case of $q=1$, the matrix in (3.1) is called a circulant matrix and will be denoted by $\mathbf{T}\left(\left[v_{n}, \ldots, v_{1}\right]\right)$.

Definition 3.1. An n-periodic signal generated from its one-period values $\left(v_{1}, \ldots, v_{n}\right)$ is said to be full rank if the circulant matrix $\mathbf{T}\left(\left[v_{n}, \ldots, v_{1}\right]\right)$ is full rank.

DEFINITION 3.2. An n-periodic signal generated from its one-period values $\left(v_{1}, \ldots, v_{n}\right)$ is said to be strongly $m$ full rank if the circulant matrices $\mathbf{T}\left(\left[v_{n}^{i}, \ldots, v_{1}^{i}\right]\right)$ are all full rank for $i=1, \ldots, m$.

Obviously, an $n$-periodic signal generated from $v=\left(v_{1}, \ldots, v_{n}\right)$ is strongly $m$ full rank if it is strongly $m+1$ full rank. An important property of circulant matrices is the following frequency-domain criterion.

Lemma 3.3 (see [15]). If $T=\mathbf{T}\left(q,\left[v_{n}, \ldots, v_{1}\right]\right)$ is a generalized circulant matrix, then the eigenvalues of $T$ are $\left\{q \gamma_{k}, k=1, \ldots, n\right\}$ and the determinant of $T$ is

$$
\operatorname{det}(T)=\prod_{k=1}^{n} q \gamma_{k}
$$

where $\gamma_{k}$ is the discrete Fourier transform (DFT) of $v_{j} q^{-\frac{j}{n}}, j=1, \ldots, n$ :

$$
\gamma_{k}=\sum_{j=1}^{n} v_{j} q^{-\frac{j}{n}} e^{-i \omega_{k} j}, \quad \omega_{k}=\frac{2 \pi k}{n}, \quad k=1, \ldots, n .
$$

Hence, $T$ is full rank if and only if $\gamma_{k} \neq 0, k=1, \ldots, n$.

For the special case of $q=1$, we have the following property.

COROLlaRY 3.4. An n-periodic signal generated from $v=\left(v_{1}, \ldots, v_{n}\right)$ is full rank if and only if its DFT

$$
\gamma_{k}=\sum_{j=1}^{n} v_{j} e^{-i \omega_{k} j}
$$

Copyright (c) by SIAM. Unauthorized reproduction of this article is prohibited. 
is nonzero at $\omega_{k}=\frac{2 \pi k}{n}, k=1, \ldots, n$.

Recall that $\left\{\gamma_{1}, \ldots, \gamma_{n}\right\}$ are the frequency samples of the $n$-periodic signal $u$. Then, Definition 3.1 may be equivalently stated as "an $n$-periodic signal $v$ is said to be full rank if its frequency samples do not contain 0." In other words, the signal contains $n$ nonzero frequency components.

For strongly full rank signals, we have the following theorem.

THEOREM 3.5. An n-periodic signal generated from $v=\left(v_{1}, \ldots, v_{n}\right)$ is strongly $m$ full rank if and only if for $l=1,2, \ldots, m$,

$$
\gamma_{k, l}=\sum_{j=1}^{n} v_{j}^{l} e^{-i \omega_{k} j}
$$

are nonzero at $\omega_{k}=\frac{2 \pi k}{n}, k=1, \ldots, n$.

Proposition 3.6. For $n=1,2$, an n-periodic signal $u$ generated from $v=$ $\left(v_{1}, \ldots, v_{n}\right)$ is strongly $m$ full rank if and only if it is full rank.

Proof. For $n=1$, by Corollary 3.4, $u$ is full rank if and only if $\gamma_{1}=v_{1} \neq 0$. By Theorem 3.5, $u$ is strongly $m$ full rank if and only if $\gamma_{1, l}=v_{1}^{l} \neq 0 \forall l$. So, $\gamma_{1} \neq 0$ is equivalent to $\gamma_{1, l} \neq 0$.

For $n=2$, by Corollary 3.4, $u$ is full rank if and only if $\gamma_{1}=v_{2}-v_{1} \neq 0$ and $\gamma_{2}=v_{2}+v_{1} \neq 0$, that is, $v_{2} \neq \pm v_{1}$. By Theorem 3.5, $u$ is strongly $m$ full rank if and only if

$$
\gamma_{1, l} \gamma_{2, l}=\left(v_{1}^{l} e^{-i \pi}+v_{2}^{l} e^{-i 2 \pi}\right)\left(v_{1}^{l} e^{-i 2 \pi}+v_{2}^{l} e^{-i 4 \pi}\right)=v_{2}^{2 l}-v_{1}^{2 l} .
$$

Thus we have $\gamma_{1} \gamma_{2}=v_{2}^{2}-v_{1}^{2} \neq 0$ if and only if $u$ is full rank.

Remark 3.7. For $n>2$, the conditions of strongly $m$ full rank may be different from the conditions of full rank. For example, for $n=3$ and $l=1, \ldots, m$,

$$
\gamma_{1, l} \gamma_{2, l} \gamma_{3, l}=\left(v_{3}^{l}+v_{2}^{l}+v_{1}^{l}\right)\left[\left(v_{2}^{l}-\frac{1}{2}\left(v_{3}^{l}+v_{1}^{l}\right)\right)^{2}+\frac{3}{4}\left(v_{3}^{l}-v_{1}^{l}\right)^{2}\right] \neq 0
$$

is not equivalent to

$$
\gamma_{1} \gamma_{2} \gamma_{3}=\left(v_{3}+v_{2}+v_{1}\right)\left[\left(v_{2}-\frac{1}{2}\left(v_{3}+v_{1}\right)\right)^{2}+\frac{3}{4}\left(v_{3}-v_{1}\right)^{2}\right] \neq 0
$$

except for $m=1$.

Definition 3.8. A 2n(m+1)-periodic signal $u$ is strongly scaled $m$ full rank if its one-period values are $\left(v, v, \rho_{1} v, \rho_{1} v, \ldots, \rho_{m} v, \rho_{m} v\right)$, where $v=\left(v_{1}, \ldots, v_{n}\right)$ is strongly $m$ full rank, and $\rho_{j} \neq 0, \rho_{j} \neq 1, j=1, \ldots, m$, and $\rho_{i} \neq \rho_{j}, i \neq j$. We use $\mathcal{U}(m)$ to denote the class of such signals.

Definition 3.9. An $n(m+1)$-periodic signal $u$ is exponentially strongly scaled $m$ full rank if its one-period values are $\left(v, q v, \ldots, q^{m} v\right)$, where $q \neq 0$ and $q \neq 1$, and $T_{j}=T_{j}\left(q^{j},\left[v_{n}^{j}, \ldots, v_{1}^{j}\right]\right)$ are all full rank for $j=1, \ldots, m$. We use $\mathcal{U}_{q}(m)$ to denote this class of input signals.

By Definition 3.9 and Lemma 3.3, we have the following.

TheOREM 3.10. An $n(m+1)$-periodic signal $u$ with one-period values $\left(v, q v, \ldots, q^{m} v\right)$ is exponentially strongly scaled $m$ full rank if $q \neq 0$ and $q \neq 1$, and if for $l=1, \ldots, m$,

$$
\gamma_{k, l}=\sum_{j=1}^{n} v_{j}^{l} q^{-\frac{j l}{n}} e^{-i \omega_{k} j}
$$

Copyright $@$ by SIAM. Unauthorized reproduction of this article is prohibited. 
are nonzero at $\omega_{k}=\frac{2 \pi k}{n}, k=1, \ldots, n$.

Remark 3.11. Definitions 3.8 and 3.9 require that $\mathbf{T}\left(q^{i},\left[v_{n}^{i}, \ldots, v_{1}^{i}\right]\right), i=1, \ldots, m$, be all full rank for $q=1$ and $q \neq 0,1$, respectively. However, since the event of singular random matrices has probability zero, if $v$ is chosen randomly, almost all $v$ will satisfy the conditions in Definitions 3.8 and 3.9, which will be shown in the following example.

Example 3.12. For $n=4, m=4, q=0.9$, and for $v=(0.5997,0.9357,0.9841,1.4559)$ generated randomly by MATLAB, $v$ is strongly 4 full rank since

$$
\begin{aligned}
\operatorname{det}\left(\mathbf{T}\left(\left[v_{4}, v_{3}, v_{2}, v_{1}\right]\right)\right) & =0.4041, \quad \operatorname{det}\left(\mathbf{T}\left(\left[v_{4}^{2}, v_{3}^{2}, v_{2}^{2}, v_{1}^{2}\right]\right)\right)=2.4823, \\
\operatorname{det}\left(\mathbf{T}\left(\left[v_{4}^{3}, v_{3}^{3}, v_{2}^{3}, v_{1}^{3}\right]\right)\right) & =7.7467, \quad \operatorname{det}\left(\mathbf{T}\left(\left[v_{4}^{4}, v_{3}^{4}, v_{2}^{4}, v_{1}^{4}\right]\right)\right)=19.8312 .
\end{aligned}
$$

Furthermore, for $q=0.9$,

$$
\begin{aligned}
\operatorname{det}\left(\mathbf{T}\left(q,\left[v_{4}, v_{3}, v_{2}, v_{1}\right]\right)\right) & =0.3796, \quad \operatorname{det}\left(\mathbf{T}\left(q^{2},\left[v_{4}^{2}, v_{3}^{2}, v_{2}^{2}, v_{1}^{2}\right]\right)\right)=1.7872, \\
\operatorname{det}\left(\mathbf{T}\left(q^{3},\left[v_{4}^{3}, v_{3}^{3}, v_{2}^{3}, v_{1}^{3}\right]\right)\right) & =4.2853, \quad \operatorname{det}\left(\mathbf{T}\left(q^{4},\left[v_{4}^{4}, v_{3}^{4}, v_{2}^{4}, v_{1}^{4}\right]\right)\right)=8.5037,
\end{aligned}
$$

and $v$ generated randomly 10000 times, it is shown that all $\mathbf{T}\left(\left[v_{4}^{i}, v_{3}^{i}, v_{2}^{i}, v_{1}^{i}\right]\right)$ and $\mathbf{T}\left(q^{i}\right.$, $\left.\left[v_{4}^{i}, v_{3}^{i}, v_{2}^{i}, v_{1}^{i}\right]\right), i=1, \ldots, 4$, are nonsingular.

4. Estimates of $\zeta$ with individual thresholds. Based on strongly scaled full rank signals, we now derive the estimation algorithms for $\zeta$ and analyze their convergence properties. To this end, estimation algorithms based on the information of individual thresholds is first investigated.

Assumption A1. The noise $\{d(k)\}$ is a sequence of independent and identically distributed (i.i.d.) random variables with finite variance. The distribution function $F(\cdot)$ of $d(1)$ is known, which is continuously differentiable and has a continuously differentiable inverse $F^{-1}(\cdot)$ and bounded density $f(\cdot)$ with $f(x) \neq 0$.

Remark 4.1. The requirements for noise distribution functions in Assumption A1 are satisfied in many typical classes of noises, such as Gaussian. Indeed, these requirements can be weakened to deal with unknown distributions similar to [22]. But, for simplicity, this paper deals with only these noises satisfying Assumption A1.

Assumption A2. The prior information on

$$
\theta=\left[a_{0}, \ldots, a_{n-1}\right]^{T} \text { and } \eta=\left[b_{0}, \ldots, b_{m-1}\right]^{T}
$$

is that $\sum_{i=0}^{n-1} a_{i} \neq 0, b_{m}=1, \eta \neq 0, \theta \in \Omega_{\theta}$, and $\eta \in \Omega_{\eta}$, where $\Omega_{\theta}$ and $\Omega_{\eta}$ are known compact sets.

The input is a scaled $2 n(m+1)$-periodic signal with one period values

$$
\left(v, v, \rho_{1} v, \rho_{1} v, \ldots, \rho_{m} v, \rho_{m} v\right),
$$

where $v=\left(v_{1}, \ldots, v_{n}\right)$ is strongly $m$ full rank.

By periodicity, $\Phi_{j}(k)=\Phi_{j}$ for $j=0, \ldots, n$, and $\Phi_{j}$ can be decomposed into $2(m+1)$ submatrices $\Phi_{j, i}, i=1, \ldots, 2(m+1)$, of dimension $n \times n$ :

$$
\Phi_{j}=\left[\Phi_{j, 1}^{T}, \Phi_{j, 2}^{T}, \ldots, \Phi_{j, 2(m+1)}^{T}\right]^{T} .
$$

Actually, for $k=1, \ldots, 2(m+1)$,

$$
\Phi_{j, k}=\left[\begin{array}{l}
\phi_{j}^{T}(k n) \\
\phi_{j}^{T}(k n+1) \\
\vdots \\
\phi_{j}^{T}(k n+n-1)
\end{array}\right] .
$$

Copyright (c) by SIAM. Unauthorized reproduction of this article is prohibited. 
Denote the $n \times n$ circulant matrix

$$
V_{0}=T([1, \ldots, 1]) \quad \text { and } \quad V_{j}=T\left(\left[v_{n}^{j}, \ldots, v_{1}^{j}\right]\right), \quad j=1, \ldots, m .
$$

Then, for $j=0, \ldots, m$, the odd-indexed block matrices satisfy the simple scaling relationship

$$
\Phi_{j, 1}=V_{j}, \quad \Phi_{j, 3}=\rho_{1}^{j} V_{j}, \ldots, \Phi_{j, 2 m+1}=\rho_{m}^{j} V_{j},
$$

and the even-indexed block matrices are

$$
\Phi_{j, 2 l}=\rho_{l-1}^{j} \mathbf{T}\left(\left(\rho_{l} / \rho_{l-1}\right)^{j},\left[v_{n}, v_{n-1}, \ldots, v_{1}\right]\right), \quad l=1, \ldots, m+1,
$$

where $\rho_{0}=\rho_{m+1}=1$.

Denote

$$
\tau_{j}=\left[\tau_{j, 1}, \ldots, \tau_{j, n}\right]^{T}=V_{j} \theta, \quad j=0, \ldots, m
$$

Then, we have

$$
\Phi_{j, 1} \theta=\tau_{j}, \quad \Phi_{j, 3} \theta=\rho_{1}^{j} \tau_{j}, \ldots, \quad \Phi_{j, 2 m+1} \theta=\rho_{m}^{j} \tau_{j} .
$$

Let

$$
\Psi_{\theta}=\left[\Phi_{0} \theta, \Phi_{1} \theta, \ldots, \Phi_{m} \theta\right]
$$

Then, from (2.5) we have

$$
Y(l)=\Psi_{\theta}\left[\eta^{T}, 1\right]^{T} \theta+D(l)=\zeta+D(l) .
$$

Remark 4.2. In $\left(v, v, \rho_{1} v, \rho_{1} v, \ldots, \rho_{m} v, \rho_{m} v\right)$, there are always two identical subsequences $\rho_{i} v, i=1, \ldots, m$, appearing consecutively. The main reason for this input structure is to generate block matrices that satisfy the above scaling relationship (4.1).

We use the following notation for elementwise vector functions. For a scalar function $g(\cdot)$ and a vector $x=\left[x_{1}, \ldots, x_{l}\right]^{T} \in \mathbb{R}^{l}$, the boldface symbol $\mathbf{g}(x)$ represents

$$
\mathbf{g}(x)=\left[g\left(x_{1}\right), \ldots, g\left(x_{l}\right)\right]^{T} \in \mathbb{R}^{l} .
$$

In addition, if $g(x)$ is invertible, $\mathbf{g}^{-1}(x)$ represents the componentwise inverse

$$
\mathbf{g}^{-1}(x)=\left[g^{-1}\left(x_{1}\right), \ldots, g^{-1}\left(x_{l}\right)\right]^{T} \in \mathbb{R}^{l} .
$$

Similarly, for $\alpha=\left[\alpha_{1}, \ldots, \alpha_{l}\right]^{T} \in \mathbb{R}^{l}$ and $c=\left[c_{1}, \ldots, c_{l}\right]^{T} \in \mathbb{R}^{l}$, we use the vector notation $\mathbf{I}_{\{\alpha \leq c\}}=\left[I_{\left\{\alpha_{1} \leq c_{1}\right\}}, \ldots, I_{\left\{\alpha_{l} \leq c_{l}\right\}}\right]^{T}$. $\mathbb{1}_{\ell}$ and $\mathbf{0}_{\ell} \in \mathbb{R}^{\ell}$ will denote column vectors with all components being 1 and 0 , respectively.

For (4.4), let

$$
\begin{aligned}
\varphi_{i}(N) & =\left[\varphi_{i, 1}(N), \ldots, \varphi_{i, 2 n(m+1)}(N)\right]^{T} \\
& =\frac{1}{N} \sum_{k=1}^{N} S_{i}(k)=\frac{1}{N} \sum_{k=1}^{N} \mathbf{I}\left\{D(k) \leq C_{i} \mathbb{1}_{2 n(m+1)}-\Psi_{\theta}\left[\eta^{T}, 1\right]^{T}\right\},
\end{aligned}
$$

which is the empirical distribution of $D(l)$ at

$$
C_{i} \mathbb{1}_{2 n(m+1)}-\zeta=C_{i} \mathbb{1}_{2 n(m+1)}-\Psi_{\theta}\left[\eta^{T}, 1\right]^{T} .
$$

Copyright (c) by SIAM. Unauthorized reproduction of this article is prohibited. 
Then, by the strong law of large numbers,

$$
\varphi_{i}(N) \rightarrow p_{i}=\mathbf{F}\left(C_{i} \mathbb{1}_{2 n(m+1)}-\Psi_{\theta}\left[\eta^{T}, 1\right]^{T}\right) \quad \text { w.p.1. }
$$

Denote $S_{i}(N)=\left[S_{i, 1}, \ldots, S_{i, 2 n(m+1)}\right]^{T}$. By Assumption A1, for each $i=1, \ldots, l$, $S_{i}(N)$ is i.i.d. Noticing that for $j=1, \ldots, 2 n(m+1), E S_{i, j}(k)=p_{i, j}=\mathbf{F}\left(C_{i}-\zeta_{j}\right)$ and

$$
E\left(S_{i, j}(k)-p_{i, j}\right)^{2}=p_{i, j}\left(1-p_{i, j}\right):=\Delta_{i, j}^{2}
$$

we have

$$
\begin{gathered}
E \varphi_{i, j}(N)=\frac{1}{N} \sum_{k=1}^{N} E S_{i, j}(k)=p_{i, j} \\
E\left(\varphi_{i, j}(N)-p_{i, j}\right)^{2}=\frac{\Delta_{i, j}^{2}}{N}
\end{gathered}
$$

Notice that $F$ is a monotone function by Assumption A1, and $\Omega_{\theta}$ and $\Omega_{\eta}$ are bounded by Assumption A2. Then, there exists $z>0$ such that

$$
z<p_{i, j}=F\left(C_{i}-\zeta_{j}\right)<1-z, \quad i=1, \ldots, l, \quad j=1, \ldots, 2 n(m+1) .
$$

Since $F(\cdot)$ is not invertible at 0 and 1 , we modify $\varphi_{i, j}$ to avoid these points. Let

$$
\xi_{i, j}(N)=\left\{\begin{array}{lll}
\varphi_{i, j}(N) & \text { if } \quad z \leq \varphi_{i, j}(N) \leq 1-z \\
z & \text { if } \quad \varphi_{i, j}(N)<z \\
1-z & \text { if } \quad \varphi_{i, j}(N)>1-z
\end{array}\right.
$$

Denote

By $\varphi_{i, j}(N) \rightarrow p_{i, j}$ w.p.1 and $z<p_{i, j}<1-z$, we have $\xi_{i, j}(N) \rightarrow p_{i, j}$ w.p.1.

$$
\xi_{i}(N)=\left[\xi_{i, 1}(N), \ldots, \xi_{i, 2 n(m+1)}(N)\right]^{T} .
$$

By Assumption A1, $F$ has a continuous inverse. Hence, for each $i=1, \ldots, l$,

$$
\begin{aligned}
\zeta_{i}(N) & =\left[\zeta_{i, 1}(N), \ldots, \zeta_{i, 2 n(m+1)}(N)\right]^{T}:=C_{i} \mathbb{1}_{2 n(m+1)}-\mathbf{F}^{-1}\left(\xi_{i}(N)\right) \\
& \rightarrow C_{i} \mathbb{1}_{2 n(m+1)}-\mathbf{F}^{-1}\left(p_{i}\right)=\Psi_{\theta}\left[\eta^{T}, 1\right]^{T}=\zeta=\left[\zeta_{1}, \ldots, \zeta_{2 n(m+1)}\right]^{T} \quad \text { w.p.1. }
\end{aligned}
$$

5. Quasi-convex combination estimates of $\boldsymbol{\zeta}$. For each $i, \zeta_{i}(N)$ is an estimate of $\zeta$ constructed by using the individual threshold $C_{i}$. They can be combined to derive a common estimate of $\zeta$. By treating the coefficients of the combination as design variables, an estimate can be obtained with minimal variance. This resulting estimate, called "quasi-convex combination estimate," is described below.

For $j=1, \ldots, 2 n(m+1)$, define $\zeta_{\cdot, j}(N)=\left[\zeta_{1, j}(N), \ldots, \zeta_{l, j}(N)\right]^{T}$ and $c_{j}(N)=$ $\left[c_{j, 1}(N), \ldots, c_{j, l}(N)\right]^{T}$ with $c_{j, 1}(N)+\cdots+c_{j, l}(N)=1$. Construct an estimate of $\zeta_{j}$ by defining

$$
\widehat{\zeta}_{j}(N)=c_{j}^{T} \zeta_{\cdot, j}(N)=\sum_{k=1}^{l} c_{j, k}(N) \zeta_{k, j}(N) .
$$

Copyright (C) by SIAM. Unauthorized reproduction of this article is prohibited. 
Suppose there exists $c_{j}=\left[c_{j, 1}, \ldots, c_{j, l}\right]^{T}$ such that $c_{j}(N) \rightarrow c_{j}$. Then $c_{j, 1}+\cdots+c_{j, l}=$ 1 , and by (4.10),

$$
\widehat{\zeta}_{j}(N)=\sum_{k=1}^{l} c_{j, k}(N) \zeta_{j, k}(N) \rightarrow \zeta_{j} \sum_{k=1}^{l} c_{j, k}=\zeta_{j} .
$$

Denote the estimation errors

$$
e_{j}(N)=\widehat{\zeta}_{j}(N)-\zeta_{j}, \quad \varepsilon_{j}(N)=\zeta_{, j}(N)-\zeta_{j} \mathbb{1}_{l},
$$

and their covariances $\sigma_{j}^{2}(N)=E e_{j}(N) e_{j}(N)^{T}, Q_{j}(N)=E \varepsilon_{j}(N) \varepsilon_{j}(N)^{T}$, respectively. Then the covariance of estimation error can be expressed in a quadratic form with respect to the variable $c_{j}$ :

$$
\begin{aligned}
\sigma_{j}^{2}(N) & :=E\left(\widehat{\zeta}_{j}(N)-\zeta_{j}\right)^{2}=E\left(\sum_{k=1}^{l} c_{j, k}(N)\left(\zeta_{k, j}(N)-\zeta_{j}\right)\right)^{2} \\
& =c_{j}^{T}(N) E \varepsilon_{j}(N) \varepsilon_{j}(N)^{T} c_{j}(N)=c_{j}^{T}(N) Q_{j}(N) c_{j}(N) .
\end{aligned}
$$

To obtain a good quasi-convex combination estimate, we choose $c_{j}$ by minimizing $\sigma_{j}^{2}(N)$ subject to the constraint $c_{j}^{T}(N) \mathbb{1}_{l}=1$, that is,

$$
c_{j}^{*}(N)=\operatorname{argmin}_{c_{j}^{T} \mathbb{1}_{l}=1} \sigma_{j}^{2}(N) .
$$

Theorem 5.1 (see [21]). Under Assumptions A1 and A2, suppose $u \in \mathcal{U}_{m}$ and that $R_{j}(N)=N Q_{j}(N)=N E \varepsilon_{j}(N) \varepsilon_{j}(N)^{T}(j=1, \ldots, 2 n(m+1))$ is positive definite. Then, the quasi-convex combination estimate can be obtained by choosing

$$
c_{j}^{*}(N)=\frac{R_{j}^{-1}(N) \mathbb{1}_{l}}{\mathbb{1}_{l}^{T} R_{j}^{-1}(N) \mathbb{1}_{l}}, \quad \widehat{\zeta}_{j}(N)=\sum_{i=1}^{l} c_{j, i}^{*} \zeta_{i, j}(N),
$$

and the minimal variance satisfies

$$
N \sigma_{j}^{2 *}(N)=\frac{1}{\mathbb{1}_{l}^{T} R_{j}^{-1}(N) \mathbb{1}_{l}} .
$$

5.1. Consistency and efficiency. From $(5.4), \widehat{\zeta}_{j}$ can be regarded as an estimate of $\zeta_{j}$. In this subsection, consistency and efficiency properties of this estimate will be analyzed.

By Assumption A1, $G(x)=F^{-1}(x)$ is continuous on $(0,1)$. As a result, $G(x)$ is bounded on the compact set $[z, 1-z]$. Since $\zeta_{i, j}(N)=C_{i}-G\left(\xi_{i, j}(N)\right) \rightarrow \zeta_{i, j}$ w.p.1, we have $\zeta_{i, j}(N) \rightarrow \zeta_{i, j}$ in probability. Furthermore, by the Lebesgue dominated convergence theorem [6, p. 99], $E \zeta_{i, j}(N) \rightarrow \zeta_{j}$. Hence,

$$
E \widehat{\zeta}_{j}(N)=E \sum_{k=1}^{l} c_{j, k}(N) \zeta_{k, j}(N) \rightarrow \zeta_{j},
$$

which means the estimate of $\zeta_{j}$ is asymptotically unbiased.

We now proceed to study efficiency of the estimator. The properties of $\xi_{i, j}(N)$ in (4.8) will first be introduced.

Copyright $@$ by SIAM. Unauthorized reproduction of this article is prohibited. 
TheOREM 5.2. Suppose $u \in \mathcal{U}(m)$. Under Assumptions A1 and A2, there exist $K_{i, j} \in(0, \infty)$ and $L_{i, j} \in(0, \infty), i=1, \ldots, l, j=1, \ldots, 2 n(m+1)$, such that

$$
P\left\{\xi_{i, j}(N) \neq \varphi_{i, j}(N)\right\} \leq K_{i, j} e^{-L_{i, j} N} .
$$

Proof. Denote $X_{i, j}=\left(S_{i, j}(1)-p_{i, j}\right) / \Delta_{i, j}$. Note that $E X_{i, j}=0$ and $E X_{i, j}^{2}=1$. By the i.i.d. assumption, taking a Taylor expansion of

$$
M_{i, j}(h, N)=\left[E \exp \left(h X_{i, j} / \sqrt{N}\right)\right]^{N}
$$

which is the moment generating function of $\sqrt{N}\left(\varphi_{i, j}(N)-p_{i, j}\right) / \Delta_{i, j}$, we obtain

$$
\begin{aligned}
M_{i, j}(h, N) & =\left[E\left[1+\frac{h X_{i, j}}{\sqrt{N}}+\frac{h^{2} X_{i, j}^{2}}{2 N}+O\left(N^{-3 / 2}\right)\right]\right]^{N} \\
& =\left[1+\frac{h^{2}}{2 N}+O\left(N^{-(3 / 2)}\right)\right]^{N}
\end{aligned}
$$

Consequently, for any $t \in \mathbb{R}$,

$$
\inf _{h} e^{-h t} M_{i, j}(h, N)=\inf _{h} e^{-h t}\left[1+\frac{h^{2}}{2 N}+O\left(N^{-(3 / 2)}\right)\right]^{N} \leq K e^{-\frac{t^{2}}{2}},
$$

where $K>0$ is a positive constant.

By means of the Chernoff bound [20, p. 326], for any $t \in\left(-\infty, p_{i, j}\right]$,

$$
\begin{aligned}
P\left\{\varphi_{i, j}(N) \leq t\right\} & =P\left\{\sum_{k=1}^{N}\left(S_{i, j}(k)-p_{i, j}\right) \leq N \frac{\left(t-p_{i, j}\right)}{\Delta_{i, j}}\right\} \\
& \leq\left\{\inf _{h}\left[e^{-\frac{h\left(t-p_{i, j}\right)}{\Delta_{i, j}}} M_{i, j}(h, N)\right]\right\}^{N},
\end{aligned}
$$

and for any $p_{i, j} \leq t<\infty$,

$$
P\left\{\varphi_{i, j}(N) \geq t\right\} \leq\left\{\inf _{h}\left[e^{-\frac{h\left(t-p_{i, j}\right)}{\Delta_{i, j}}} M_{i, j}(h, N)\right]\right\}^{N} .
$$

Considering

$$
P\left\{\xi_{i, j}(N) \neq \varphi_{i, j}(N)\right\}=P\left(\varphi_{i, j}(N) \leq z\right)+P\left(\varphi_{i, j}(N) \geq 1-z\right)
$$

and (5.7)-(5.9), we have that (5.6) is true.

THEOREM 5.3. Under the conditions of Theorem 5.2, we have

$$
\begin{gathered}
N E\left(\xi_{i, j}(N)-p_{i, j}\right)^{2} \rightarrow \Delta_{i, j}^{2}, \quad N \rightarrow \infty, \\
N E\left|\left(\xi_{i, j}(N)-p_{i, j}\right)\right|^{m} \rightarrow 0, \quad N \rightarrow \infty, \quad m=3,4, \ldots
\end{gathered}
$$

Proof. (i) By Theorem 5.2, there exist $K_{i, j} \in(0, \infty)$ and $L_{i, j} \in(0, \infty)$ such that

$$
E N\left(\xi_{i, j}(N)-\varphi_{i, j}(N)\right)^{2} \leq N z P\left\{\xi_{i, j}(N) \neq \varphi_{i, j}(N)\right\} \leq z K_{i, j} N e^{-L_{i, j} N} \rightarrow 0
$$

Copyright (C) by SIAM. Unauthorized reproduction of this article is prohibited. 
This, together with

$$
\begin{array}{rl}
E & N\left(\varphi_{i, j}(N)-p_{i, j}\right)\left(\xi_{i, j}(N)-\varphi_{i, j}(N)\right) \\
& \leq \sqrt{E N\left(\varphi_{i, j}(N)-p_{i, j}\right)^{2} E N\left(\xi_{i, j}(N)-\varphi_{i, j}(N)\right)^{2}} \\
& =\Delta_{i, j} \sqrt{E N\left(\xi_{i, j}(N)-\varphi_{i, j}(N)\right)^{2}},
\end{array}
$$

implies that

$$
\begin{aligned}
& E N\left(\xi_{i, j}(N)-p_{i, j}\right)^{2}-E N\left(\varphi_{i, j}(N)-p_{i, j}\right)^{2} \\
& \quad=2 E N\left(\varphi_{i, j}(N)-p_{i, j}\right)\left(\xi_{i, j}(N)-\varphi_{i, j}(N)\right)+E N\left(\xi_{i, j}(N)-\varphi_{i, j}(N)\right)^{2} \\
& \quad \rightarrow 0, \quad N \rightarrow \infty .
\end{aligned}
$$

Thus, by (4.7) we get (5.10).

(ii) Similarly, for $m=3,4, \ldots$, one can get

$$
N E\left|\left(\xi_{i, j}(N)-p_{i, j}\right)\right|^{m}-N E\left|\left(\varphi_{i, j}(N)-p_{i, j}\right)\right|^{m} \rightarrow 0
$$

By Hölder's inequality,

$$
N E\left|\varphi_{i, j}(N)-p_{i, j}\right|^{m} \leq \Delta_{i, j} \sqrt{N E\left(\varphi_{i, j}(N)-p_{i, j}\right)^{2(m-1)}} .
$$

Notice that for each $i$ and $j, S_{i, j}(k)$ is i.i.d. Then, we have

$$
\begin{aligned}
N E\left(\varphi_{i, j}(N)-p_{i, j}\right)^{2(m-1)} & =N E\left[\frac{1}{N} \sum_{k=1}^{N}\left(S_{i, j}(k)-p_{i, j}\right)\right]^{2(m-1)} \\
& =N^{-2(m-2)} E\left(S_{i, j}(1)-p_{i, j}\right)^{2(m-1)} \\
& \leq N^{-2(m-2)}
\end{aligned}
$$

which, together with (5.13), results in

$$
N E\left|\varphi_{i, j}(N)-p_{i, j}\right|^{m} \leq \Delta_{i, j} N^{-(m-2)} \rightarrow 0 .
$$

Hence, (5.11) is obtained.

From (5.5), the covariance of the estimation $\widehat{\zeta}_{j}(N)$ is decided by $R_{j}(N)$.

TheOrem 5.4. Suppose $u \in \mathcal{U}(m)$. If, in addition to Assumptions A1 and A2, the density function $f(x)$ is continuously differentiable, then

$$
R_{j}(N):=N Q_{j}(N)=N E \varepsilon_{j}(N) \varepsilon_{j}(N)^{T} \rightarrow \Lambda_{j} W_{j} \Lambda_{j}:=R_{j}, \quad N \rightarrow \infty,
$$

where $\varepsilon_{j}(N)=\zeta_{\cdot, j}(N)-\zeta_{j} \mathbb{1}_{l}, \Lambda_{j}=\operatorname{diag}^{-1}\left\{f\left(C_{1}-\zeta_{j}\right), \ldots, f\left(C_{l}-\zeta_{j}\right)\right\}$, and

$$
W_{j}=\left(\begin{array}{cccc}
p_{1, j}\left(1-p_{1, j}\right) & p_{1, j}\left(1-p_{2, j}\right) & \cdots & p_{1, j}\left(1-p_{l, j}\right) \\
p_{1, j}\left(1-p_{2, j}\right) & p_{2, j}\left(1-p_{2, j}\right) & & p_{2, j}\left(1-p_{l, j}\right) \\
\vdots & & \ddots & \vdots \\
p_{1, j}\left(1-p_{l, j}\right) & p_{2, j}\left(1-p_{l, j}\right) & \cdots & p_{l, j}\left(1-p_{l, j}\right)
\end{array}\right)
$$

Copyright (c) by SIAM. Unauthorized reproduction of this article is prohibited. 
Proof. Denote by $\varepsilon_{j, i}(N)$ the $i$ th component of $\varepsilon_{j}(N), G^{\prime}(x)=d G(x) / d x$, and $G^{\prime \prime}(x)=d G^{\prime}(x) / d x$. Then

$$
\begin{aligned}
& G^{\prime}(x)=\frac{d G(x)}{d x}=\frac{d G(x)}{d F(G(x))}=\frac{1}{f(G(x))}, \\
& G^{\prime \prime}(x)=\frac{d G^{\prime}(x)}{d x}=-\frac{1}{f^{2}(G(x))} f^{\prime}(G(x)) G^{\prime}(x) .
\end{aligned}
$$

Since $f^{\prime}(x)$ is continuous by Assumption A1, both $G^{\prime}(x)$ and $G^{\prime \prime}(x)$ are continuous, and hence bounded in $[z, 1-z]$. Let $\beta_{i, j}=\sup _{x \in[z, 1-z]}\left\{\left|G^{\prime}(x)\right|\right\}$ and $\gamma_{i, j}=$ $\sup _{x \in[z, 1-z]}\left\{\left|G^{\prime \prime}(x)\right|\right\}$. Then, there exists a number $\lambda_{i, j}(N)$ between $p_{i, j}$ and $\xi_{i, j}(N)$ such that

$$
\begin{aligned}
\varepsilon_{j, i} & =\zeta_{i, j}(N)-\zeta_{j}=G\left(\xi_{i, j}(N)\right)-G\left(p_{i, j}\right) \\
& =G^{\prime}\left(p_{i, j}\right)\left(\xi_{i, j}(N)-p_{i, j}\right)+\frac{1}{2} G^{\prime \prime}\left(\lambda_{i, j}(N)\right)\left(\xi_{i, j}(N)-p_{i, j}\right)^{2} .
\end{aligned}
$$

This implies that for $i, k=1, \ldots, l$,

$$
\begin{aligned}
N E \varepsilon_{j, i}(N) \varepsilon_{j, k}(N)= & N E\left(\zeta_{i, j}(N)-\zeta_{j}\right)\left(\zeta_{k, j}(N)-\zeta_{j}\right) \\
= & N G^{\prime}\left(p_{i, j}\right) G^{\prime}\left(p_{k, j}\right) E\left(\xi_{i, j}(N)-p_{i, j}\right)\left(\xi_{k, j}(N)-p_{k, j}\right) \\
& +N E G^{\prime}\left(p_{i, j}\right)\left(\xi_{i, j}(N)-p_{i, j}\right)\left(\xi_{k, j}(N)-p_{k, j}\right)^{2} G^{\prime \prime}\left(\lambda_{k, j}\right) \\
& +N E G^{\prime \prime}\left(\lambda_{i, j}\right)\left(\xi_{i, j}(N)-p_{i, j}\right)^{2}\left(\xi_{k, j}(N)-p_{k, j}\right) G^{\prime}\left(p_{k, j}\right) \\
& +N E G^{\prime \prime}\left(\lambda_{i, j}\right)\left(\xi_{i, j}(N)-p_{i, j}\right)^{2}\left(\xi_{k, j}(N)-p_{k, j}\right)^{2} G^{\prime}\left(\lambda_{k, j}\right) .
\end{aligned}
$$

By Hölder's inequality and Theorem 5.3, we have

$$
\begin{aligned}
& \left|N E G^{\prime}\left(p_{i, j}\right)\left(\xi_{i, j}(N)-p_{i, j}\right)\left(\xi_{k, j}(N)-p_{k, j}\right)^{2} G^{\prime \prime}\left(\lambda_{k, j}\right)\right| \\
& \quad \leq \beta_{i, j} \gamma_{i, j} \sqrt{N E\left(\xi_{i, j}(N)-p_{i, j}\right)^{2} N E\left(\xi_{k, j}(N)-p_{k, j}\right)^{4}} \\
& \quad \leq \beta_{i, j} \gamma_{i, j} \Delta_{i, j} \sqrt{N E\left(\xi_{k, j}(N)-p_{k, j}\right)^{4}} \rightarrow 0 .
\end{aligned}
$$

Similarly,

$$
\begin{gathered}
\left|N E G^{\prime \prime}\left(\lambda_{i, j}\right)\left(\xi_{i, j}(N)-p_{i, j}\right)^{2}\left(\xi_{k, j}(N)-p_{k, j}\right) G^{\prime}\left(p_{k, j}\right)\right| \rightarrow 0, \\
\left|N E G^{\prime \prime}\left(\lambda_{i, j}\right)\left(\xi_{i, j}(N)-p_{i, j}\right)^{2}\left(\xi_{k, j}(N)-p_{k, j}\right)^{2} G^{\prime}\left(\lambda_{k, j}\right)\right| \rightarrow 0 .
\end{gathered}
$$

Thus, similar to (5.12), we have

$N\left[E\left(\xi_{i, j}(N)-p_{i, j}\right)\left(\xi_{k, j}(N)-p_{k, j}\right)-E\left(\varphi_{i, j}(N)-p_{i, j}\right)\left(\varphi_{k, j}(N)-p_{k, j}\right)\right] \rightarrow 0$.

Since $d(k), k=1,2, \ldots$, are i.i.d. random variables,

$$
\begin{aligned}
& N E\left(\varphi_{i, j}(N)-p_{i, j}\right)\left(\varphi_{k, j}(N)-p_{k, j}\right) \\
& =\frac{1}{N} E\left[\left(\sum_{l_{1}=1}^{N} I\left\{d\left(l_{1}\right) \leq F^{-1}\left(p_{i, j}\right)\right\}-p_{i, j}\right)\left(\sum_{l_{2}=1}^{N} I\left\{d\left(l_{2}\right) \leq F^{-1}\left(p_{k, j}\right)\right\}-p_{k, j}\right)\right] \\
& =\frac{1}{N} E \sum_{l_{1}=1}^{N} I\left\{d\left(l_{1}\right) \leq F^{-1}\left(p_{i, j}\right)\right\} I\left\{d\left(l_{1}\right) \leq F^{-1}\left(p_{k, j}\right)\right\}-p_{i, j} p_{k, j} \\
& =p_{\min \{i, k\}, j}-p_{i, j} p_{k, j}
\end{aligned}
$$

Copyright (C) by SIAM. Unauthorized reproduction of this article is prohibited. 
and

$$
G^{\prime}\left(p_{i, j}\right)=\frac{1}{f\left(G\left(p_{i, j}\right)\right)}=\frac{1}{f\left(C_{i}-\zeta_{j}\right)} .
$$

Therefore, (5.14) follows from (5.16)-(5.22).

Proposition 5.5. $R_{j}, j=1, \ldots, 2 n(m+1)$, defined by $(5.14)$, is positive definite, and

$$
\mathbb{1}_{l}^{T} R_{j}^{-1} \mathbb{1}_{l}=\sum_{k=1}^{l+1} \frac{h_{k, j}^{2}}{\widetilde{p}_{k, j}},
$$

where $\widetilde{p}_{i, j}=F\left(C_{i}-\zeta_{j}\right)-F\left(C_{i-1}-\zeta_{j}\right), h_{i, j}=f\left(C_{i-1}-\zeta_{j}\right)-f\left(C_{i}-\zeta_{j}\right)$ for $C_{0}=-\infty$, $C_{l+1}=\infty$.

Proof. Since $R_{j}(N)=N E \varepsilon_{j}(N) \varepsilon_{j}(N)^{T} \geq 0$, so is $R_{j}$. Noting $R_{j}=\Lambda_{j} W_{j} \Lambda_{j}$, $\Lambda_{j}=\operatorname{diag}^{-1}\left\{f\left(C_{1}-\zeta_{j}\right), \ldots, f\left(C_{l}-\zeta_{j}\right)\right\}$, and $f\left(C_{i}-\zeta_{j}\right)>0, i=1, \ldots, l$, we need only show that $W_{j}$ is positive definite.

From (5.15),

$$
\begin{aligned}
\operatorname{det}\left(W_{j}\right) & =\left|\begin{array}{cccc}
p_{1, j}\left(1-p_{1, j}\right) & p_{1, j}\left(1-p_{2, j}\right) & \cdots & p_{1, j}\left(1-p_{l, j}\right) \\
p_{1, j}\left(1-p_{2, j}\right) & p_{2, j}\left(1-p_{2, j}\right) & & p_{2, j}\left(1-p_{l, j}\right) \\
\vdots & & \ddots & \vdots \\
p_{1, j}\left(1-p_{l, j}\right) & p_{2, j}\left(1-p_{l, j}\right) & \cdots & p_{l, j}\left(1-p_{l, j}\right)
\end{array}\right| \\
& =p_{1, j}\left|\begin{array}{cccc}
1-p_{1, j} & p_{1, j}-p_{2, j} & \cdots & p_{1, j}-p_{l, j} \\
1-p_{2, j} & 0 & & p_{2, j}-p_{l, j} \\
\vdots & & \ddots & \vdots \\
1-p_{l, j} & 0 & \cdots & 0
\end{array}\right| \\
& =p_{1, j}\left(p_{1, j}-p_{2, j}\right) \cdots\left(p_{l, j}-p_{l-1, j}\right)\left(1-p_{l, j}\right) \neq 0 .
\end{aligned}
$$

Thus, $R_{j}>0$.

Furthermore, by [21, Lemma 7], $\mathbb{1}^{T} R_{j}^{-1} \mathbb{1}=\sum_{k=1}^{l+1} \frac{h_{k, j}^{2}}{\widetilde{p}_{k, j}}$. Thus, the second equation of (5.5) is also true.

Lemma 5.6 (see [21]). The Cramér-Rao lower bound for estimating $\zeta_{j}$ based on $\{s(k)\}$ is

$$
\sigma_{C R, j}^{2}(N)=\left(N \sum_{j=1}^{l+1} \frac{h_{i, j}^{2}}{\widetilde{p}_{i, j}}\right)^{-1}
$$

The above algorithms are asymptotically efficient based on the following theorem. ThEOREM 5.7. Under the conditions of Theorem 5.4, for $j=1, \ldots, 2 n(m+1)$,

$$
\lim _{N \rightarrow \infty} N\left(\sigma_{j}^{2 *}(N)-\sigma_{C R, j}^{2}(N)\right)=0, \quad N \rightarrow \infty .
$$

Proof. This theorem can be proved directly by Theorem 5.4, Proposition 5.5, and Lemma 5.6.

Remark 5.8. Theorem 5.7 confirms that the covariance of estimation error can be described by $\sigma_{C R, j}^{2}(N)$. Noticing that the effect of inputs, noises, and thresholds is only on $\sum_{j=1}^{l+1} \frac{h_{i, j}^{2}}{\widetilde{p}_{i, j}}$ by $(5.24)$, we can see that the covariance of estimation error has a convergence rate of $\frac{1}{N}$.

Copyright (c) by SIAM. Unauthorized reproduction of this article is prohibited. 
5.2. Recursive quasi-convex combination estimates. Since $\sigma_{j}^{2}(N)=$ $E \varepsilon_{j}(N) \varepsilon_{j}^{T}(N)$ contains an unknown parameter $\zeta_{j}$, it cannot be directly computed. As a result, the quasi-convex combination estimate $\zeta_{j}(N)$ in (5.4) cannot be computed. In this subsection, we will derive computable estimates. The basic idea is to employ a recursive structure in which the unknown $\zeta_{j}$ is replaced by the current estimate $\widehat{\zeta}_{j}(N)$. Convergence of the algorithms will be established.

For $i=1, \ldots, l$ and $j=1, \ldots, 2 n(m+1)$, let $\xi_{i}(0)=\mathbf{0}_{2 n(m+1)}, \widehat{c}_{j}(0)=\mathbf{0}_{l}$, $\widehat{R}_{j}(0)=\mathbf{0}_{l \times l}$, and $\widehat{\zeta}_{j}(0)=\mathbf{0}_{2 n(m+1)}$. Suppose that at step $N-1(N \geq 1), \xi_{i}(N-1)$, $c_{j}(N-1)$, and $\widehat{R}_{j}(N-1)$ have been obtained. Then the estimation algorithms can be constructed as follows.

(i) Calculate the sample distribution values

$$
\xi_{i}(N)=\frac{1}{N} S_{i}(N)+\frac{N-1}{N} \xi_{i}(N-1) .
$$

(ii) Calculate the data points

$$
\zeta_{i}(N)=F^{-1}\left(\xi_{i}(N)\right)
$$

Let

$$
\zeta_{\cdot, j}(N)=\left[\zeta_{1, j}(N), \ldots, \zeta_{l, j}(N)\right]^{T}, \quad j=1, \ldots, 2 n(m+1) .
$$

(iii) Calculate each covariance estimate $R_{j}(N)$.

Let $p_{i, j}(N)=F\left(C_{i}-\tilde{\zeta}_{j}(N-1)\right), \widehat{\Lambda}_{j}(N)=\operatorname{diag}^{-1}\left\{f\left(p_{1, j}(N)\right), \ldots, f\left(p_{l, j}(N)\right)\right\}$ and

$$
W_{j}(N)=\left(\begin{array}{ccc}
p_{1, j}(N)\left(1-p_{1, j}(N)\right) & \cdots & p_{1, j}(N)\left(1-p_{l, j}(N)\right) \\
\vdots & \ddots & \vdots \\
p_{1, j}(N)\left(1-p_{l, j}(N)\right) & \cdots & p_{l, j}(N)\left(1-p_{l, j}(N)\right)
\end{array}\right) .
$$

Calculate $R_{j}(N)$ by

$$
\widehat{R}_{j}(N)=\widehat{\Lambda}_{j}(N) W_{j}(N) \widehat{\Lambda}_{j}(N)
$$

(iv) If $\widehat{R}_{j}(N)$ is nonsingular, then let

$$
\widehat{c}_{j}(N)=\frac{\widehat{R}_{j}^{-1}(N) \mathbb{1}}{\mathbb{1}^{T} \widehat{R}_{j}^{-1}(N) \mathbb{1}},
$$

and compute

$$
\widehat{\zeta}_{j}(N)=\widehat{c}_{j}^{T}(N)\left(\left[C_{1}, \ldots, C_{l}\right]^{T}-\zeta_{, j}(N)\right) .
$$

Otherwise, $\widehat{\zeta}_{j}(N)=\widehat{\zeta}_{j}(N-1)$.

(v) Let $\widehat{\zeta}(N)=\left[\widehat{\zeta}_{1}(N), \ldots, \widehat{\zeta}_{2 n(m+1)}(N)\right]^{T}$. Go to step (i).

This algorithm depends only on sample paths. At each step, it minimizes estimation variance based on the most recent information on the unknown parameter. In addition, the following asymptotic properties hold.

Copyright ( by SIAM. Unauthorized reproduction of this article is prohibited. 
THEOREM 5.9. Under the condition of Theorems 5.4, for $j=1, \ldots, 2 n(m+1)$ the above recursive algorithms have the following properties:

$$
\begin{aligned}
\lim _{N \rightarrow \infty} \widehat{\zeta}_{j}(N) & =\zeta_{j} \quad \text { w.p.1, } \\
\lim _{N \rightarrow \infty} \widehat{R}_{j}(N) & =R_{j} \quad \text { w.p.1, } \\
\lim _{N \rightarrow \infty} N E\left(\widehat{\zeta}_{j}(N)-\zeta_{j}\right)^{2} & =\frac{1}{\mathbb{1}^{T} R_{j}^{-1} \mathbb{1}} \quad \text { w.p.1. }
\end{aligned}
$$

Proof. $\xi_{i}(N)$ can be written as

$$
\xi_{i}(N)=\frac{1}{N} \sum_{k=1}^{N} S_{i}(K) .
$$

The well-known Glivenko-Cantelli theorem [3, p. 103] implies that

$$
\xi_{i}(N) \rightarrow F\left(C_{i} \mathbb{1}_{2 n(m+1)}-\zeta\right) \quad \text { w.p.1, }
$$

and the convergence is uniform in $C_{i} \mathbb{1}_{2 n(m+1)}-\zeta$. Since $F(\cdot)$ and $F^{-1}(\cdot)$ are both continuous,

$$
\begin{aligned}
\zeta_{i}(N) & =C_{i} \mathbb{1}_{2 n(m+1)}-\mathbf{F}^{-1}\left(\xi_{i}(N)\right) \\
& \rightarrow C_{i} \mathbb{1}_{2 n(m+1)}-\mathbf{F}^{-1}\left(\mathbf{F}\left(C_{i} \mathbb{1}_{2 n(m+1)}-\zeta\right)\right)=\zeta \quad \text { w.p.1 } \quad \text { as } N \rightarrow \infty
\end{aligned}
$$

Thus, the quasi-convex combination $\widehat{\zeta}_{j}(N)$ converges to $\zeta$ w.p.1. That is, (5.25) holds.

By Assumption A1, $F(\cdot)$ and $f(\cdot)$ are both continuous. Hence, $\widehat{\Lambda}_{j}(N) \rightarrow \Lambda_{j}$ and $W_{j}(N) \rightarrow W_{j}$. As a result, (5.26) holds, and by (5.5),

$$
E\left(\widehat{\zeta}_{j}(N)-\zeta_{j}\right)^{2}=\frac{1}{\mathbb{1}_{l}^{T} \widehat{R}_{j}^{-1}(N) \mathbb{1}_{l}} \rightarrow \frac{1}{\mathbb{1}_{l}^{T} R_{j}^{-1} \mathbb{1}_{l}},
$$

which results in (5.27).

6. Estimation of system parameters. Identification algorithms of the system parameters will be constructed based on the estimate of $\zeta$. The parameters of linear part are first estimated, based on which the nonlinearity is identified.

\subsection{Identifiability of the unknown parameters.}

Theorem 6.1. Suppose $u \in \mathcal{U}(m)$. Then,

$$
\Psi_{\theta}\left[\eta^{T}, 1\right]^{T}=\zeta
$$

has a unique solution $\left(\theta^{*}, \eta^{*}\right)$.

Proof. (i) Calculation of $\theta^{*}$. By the first component of (4.10), we have $\zeta=$ $\left[\zeta_{1}, \ldots, \zeta_{2 n(m+1)}\right]^{T}$ and

$$
b_{0} \tau_{0,1}+b_{1} \tau_{1,1}+\cdots+b_{m} \tau_{m, 1}=\zeta_{1} .
$$

From (4.3), the $2 i n+1(i=1, \ldots, m)$ component of (4.10) turns out to be

$$
b_{0} \tau_{0,1}+\rho_{i} b_{1} \tau_{1,1}+\cdots+\rho_{i}^{m} b_{m} \tau_{m, 1}=\zeta_{2 i n+1},
$$

Copyright $@$ by SIAM. Unauthorized reproduction of this article is prohibited. 
or equivalently,

$$
\Re\left(\begin{array}{c}
b_{0} \tau_{0,1} \\
b_{1} \tau_{1,1} \\
\vdots \\
b_{m} \tau_{m, 1}
\end{array}\right)=\left(\begin{array}{c}
\zeta_{1} \\
\zeta_{2 n+1} \\
\vdots \\
\zeta_{2 m n+1}
\end{array}\right)
$$

where

$$
\Re=\left(\begin{array}{cccc}
1 & 1 & \cdots & 1 \\
1 & \rho_{1} & \cdots & \rho_{1}^{m} \\
\vdots & \cdots & \cdots & \vdots \\
1 & \rho_{m} & \cdots & \rho_{m}^{m}
\end{array}\right) .
$$

Since $\rho_{j} \neq 0, \rho_{j} \neq 1, j=1, \ldots, m$, and $\rho_{i} \neq \rho_{j}$, the determinant of the Vandermonde matrix is

$$
\operatorname{det} \Re=\prod_{0 \leq i<j \leq m-1}\left(\rho_{j}-\rho_{i}\right) \neq 0 \quad \text { with } \quad \rho_{0}=1 .
$$

Hence, $b_{j} \tau_{j, 1}, j=0, \ldots, m$, can be solved by

$$
\left(\begin{array}{c}
b_{0} \tau_{0,1} \\
b_{1} \tau_{1,1} \\
\vdots \\
b_{m} \tau_{m, 1}
\end{array}\right)=\Re^{-1}\left(\begin{array}{c}
\zeta_{1} \\
\zeta_{2 n+1} \\
\vdots \\
\zeta_{2 m n+1}
\end{array}\right) .
$$

Similarly, we have

$$
\Gamma=\Re^{-1} \Xi
$$

where

$$
\begin{aligned}
& \Gamma=\left(\begin{array}{cccc}
b_{0} \tau_{0,1} & b_{0} \tau_{0,2} & \cdots & b_{0} \tau_{0, n} \\
b_{1} \tau_{1,1} & b_{1} \tau_{1,2} & \cdots & b_{1} \tau_{1, n} \\
& & \vdots & \\
& & & \\
b_{m} \tau_{m, 1} & b_{m} \tau_{m, 2} & \cdots & b_{m} \tau_{m, n}
\end{array}\right),
\end{aligned}
$$

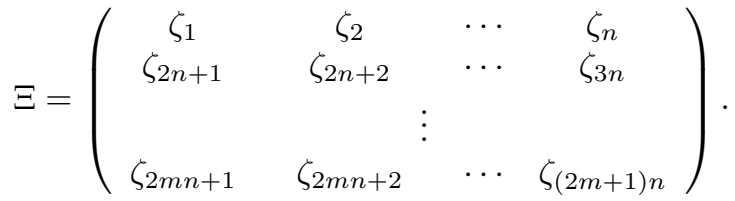

Denote $r_{i}$ as the $i$ th column of $\left(\Re^{-1}\right)^{T}$. Then, by $b_{m}=1$ we have

$$
\tau_{m}=\left[\tau_{m, 1}, \ldots, \tau_{m, n}\right]^{T}=\Xi^{T} r_{m} .
$$

Notice that $u \in \mathcal{U}(m)$ implies that $V_{m}$ is full rank. Then, by (4.2) one can get $\theta^{*}=V_{m}^{-1} \tau_{m}$.

(ii) Calculation of $\eta^{*}$. By Assumption A2, $\sum_{i=0}^{n-1} a_{i} \neq 0$ or $V_{0} \theta \neq \mathbf{0}_{n}$. For $u \in \mathcal{U}(m)$ and $j=1, \ldots, m, V_{j}=\mathbf{T}\left(\left[v_{n}^{j}, \ldots, v_{1}^{j}\right]\right)$ is full rank by Definition 3.2, and 
so $V_{j} \theta \neq \mathbf{0}_{n}$. Thus, for each $j=0, \ldots, m, \tau_{j}=V_{j} \theta$ has a nonzero component $\tau_{j, i_{j}^{*}}$. For any given positive integer $k$ and $j=1, \ldots, k$, let $\beta_{j}(k)$ be a $k$-dimensional vector with all components being zero, except the $j$ th which is 1 , that is,

$$
\beta_{j}(k)=[\underbrace{0, \ldots, 0}_{j-1}, 1, \underbrace{0, \ldots, 0}_{k-j}]^{T} .
$$

Then, from (6.2) we have

$$
b_{j} \tau_{j, i_{j}^{*}}=\beta_{j}^{T}(m+1) \Re^{-1} \Xi \beta_{i_{j}^{*}}(n), \quad j=0, \ldots, m,
$$

which gives $b_{j}, j=0, \ldots, m$, since $\tau_{j, i_{j}^{*}}$ can be calculated from $V_{j}$ and $\theta^{*}$ via (4.2). Thus, $\eta^{*}$ is obtained.

A particular choice of the scaling factors $\rho_{j}$ is $\rho_{j}=q^{j}, j=0,1, \ldots, m$, for some $q \neq 0$ and $q \neq 1$. In this case, the period of input $u$ can be shortened to $n(m+1)$ under a slightly different condition.

6.2. Identification algorithms and convergence properties. The vector

$$
\zeta(N)=\left[\zeta_{0}(N), \ldots, \zeta_{2 n(m+1)-1}(N)\right]^{T}
$$

in (4.9) has $2 n(m+1)$ components for a strongly scaled $m$ full rank signal $u \in \mathcal{U}(m)$.

Let

$$
\begin{gathered}
V_{m}=\mathbf{T}\left(\left[u_{n}^{m}, \ldots, u_{1}^{m}\right]\right), \\
\Xi(N)=\left(\begin{array}{cccc}
\zeta_{1}(N) & \zeta_{2}(N) & \ldots & \left.r_{m}\right]:=\left(\Re^{T}\right)^{-1}, \\
\zeta_{2 n+1}(N) & \zeta_{2 n+2}(N) & \cdots & \zeta_{n}(N) \\
& \vdots & & \\
\zeta_{2 m n+1}(N) & \zeta_{2 m n+2}(N) & \cdots & \zeta_{(2 m+1) n}(N)
\end{array}\right) .
\end{gathered}
$$

Then, we have the following identification algorithm:

(i) Estimation of $\theta$. The estimate of $\theta$ is taken as

$$
\theta(N)=V_{m}^{-1} \Xi^{T}(N) r_{m}
$$

(ii) Estimation of $\eta$. Let $b_{j}(0)=0$ and

$$
b_{j}(N)= \begin{cases}{\left[\zeta_{i_{j}^{*}(N)}, \zeta_{2 n+i_{j}^{*}(N)}, \ldots, \zeta_{2 m n+i_{j}^{*}(N)}\right] r_{i_{j}^{*}(N)} / \tau_{j, i_{j}^{*}}(N),} & \tau_{j, i_{j}^{*}(N)} \neq 0, \\ b_{j}(N-1), & \tau_{j, i_{j}^{*}(N)}=0,\end{cases}
$$

where

$$
i_{j}^{*}(N)=\min \left\{\operatorname{argmax}_{1 \leq i \leq n}\left|\tau_{j, i}(N)\right|\right\}, \quad j=0,1, \ldots, m-1,
$$

$r_{i_{j}^{*}(N)}$ is the $i_{j}^{*}(N)$ th column of $\left(\Re^{T}\right)^{-1}$, and $\tau_{j, i_{j}^{*}}(N)$ is the $i_{j}^{*}(N)$ th component of $\tau_{j}(N)=V_{j} \theta(N)$. Then, an estimate of $\eta$ is

$$
\eta(N)=\left[b_{0}(N), \ldots, b_{m-1}(N)\right]^{T} .
$$

Copyright $@$ by SIAM. Unauthorized reproduction of this article is prohibited. 
Theorem 6.2. Suppose $u \in \mathcal{U}(m)$. Then, under Assumptions A1 and A2,

$$
\theta(N) \rightarrow \theta, \eta(N) \rightarrow \eta \quad \text { w.p.1 as } N \rightarrow \infty
$$

Proof. By (4.10), $\zeta(N) \rightarrow \zeta$ w.p.1. as $N \rightarrow \infty$. So,

$$
\theta(N)=V_{m}^{-1} \Xi^{T}(N) r_{m} \rightarrow V_{m}^{-1} \Xi^{T} r_{m}=\theta,
$$

which in turn leads to

$$
\tau_{j}(N)=\left[\tau_{j, 1}(N), \ldots, \tau_{j, n}(N)\right]^{T}:=V_{j} \theta(N) \rightarrow V_{j} \theta=\tau_{j} \quad \text { w.p.1 }
$$

and $\tau_{j, i}(N) \rightarrow \tau_{j, i}$ w.p.1 for $i=1, \ldots, n$. Thus, for $j=0, \ldots, m-1$, we have

$$
i_{j}^{*}(N)=\min \left\{\operatorname{argmax}_{1 \leq i \leq n}\left|\tau_{j, i}(N)\right|\right\} \rightarrow \min \left\{\operatorname{argmax}_{1 \leq i \leq n}\left|\tau_{j, i}\right|\right\}:=i_{j}^{*}
$$

and

$$
\tau_{j, i_{j}^{*}}(N) \rightarrow \tau_{j, i_{j}^{*}} \neq 0
$$

This means that with probability 1 , there exists $N_{0}>0$ such that $\tau_{j, i_{j}^{*}}(N) \neq 0$ $\forall N \geq N_{0}$.

Let

$$
b_{j}(N)=\left[\zeta_{i_{j}^{*}(N)}, \zeta_{2 n+i_{j}^{*}(N)}, \ldots, \zeta_{2 m n+i_{j}^{*}(N)}\right] r_{i_{j}^{*}(N)} / \tau_{j, i_{j}^{*}}(N) .
$$

Then by

$$
b_{j}(N) \tau_{j, i_{j}^{*}}(N) \rightarrow\left[\zeta_{i_{j}^{*}}, \zeta_{2 n+i_{j}^{*}}, \ldots, \zeta_{2 m n+i_{j}^{*}}\right] r_{i_{j}^{*}}=b_{j} \tau_{j, i_{j}^{*}},
$$

we have $b_{j}(N) \rightarrow b_{j}$ for $j=0, \ldots, m-1$. Hence, $\eta(N) \rightarrow \eta$ w.p. 1 as $N \rightarrow \infty$.

6.3. Algorithms under exponentially scaled inputs. Let $u$ be $n(m+2)$ periodic; with one-period values $\left(v, q v, \ldots, q^{m} v, q^{m+1} v\right), \zeta^{e}(N)=\left[\zeta_{1}^{e}(N), \ldots, \zeta_{n}(m+1)^{e}(N)\right]$ it can be estimated by the algorithms in section 5 , with dimension changed from $2 n(m+1)$ to $n(m+2)$, and we have

$$
\zeta^{e}(N) \rightarrow \zeta^{e}=\sum_{j=0}^{m} b_{j} \Phi_{j}^{e} \theta .
$$

Partition $\Phi_{j}^{e}$ into $(m+2)$ submatrices $\Phi_{j, i}^{e}, i=1, \ldots, m+2$, of dimension $n \times n$ :

$$
\Phi_{j}^{e}=\left[\left(\Phi_{j, 1}^{e}\right)^{T},\left(\Phi_{j, 2}^{e}\right)^{T}, \ldots,\left(\Phi_{j, m+2}^{e}\right)^{T}\right]^{T} .
$$

If $u \in \mathcal{U}_{q}(m)$, then it can be directly verified that

$$
\Phi_{j, l+1}^{e}=q^{j l} V_{j}^{e}, \quad l=0,1, \ldots, m, \quad \text { and } \quad \Phi_{j, m+2}^{e}=q^{j(m+2)} \mathbf{T}\left(q^{-j(m+2)},\left[v_{n}, \ldots, v_{1}\right]\right),
$$

where $V_{j}^{e}=T\left(q^{j},\left[v_{n}^{j}, \ldots, v_{1}^{j}\right]\right)$. With this notation, we have the following result, whose proof is similar to that of Theorem 6.1 , and hence is omitted.

Theorem 6.3. Suppose $u \in \mathcal{U}_{q}(m)$. Then, under Assumptions A1 and A2,

$$
\Psi_{\theta}^{e}\left[\eta^{T}, 1\right]^{T}=\zeta^{e}
$$

Copyright (c) by SIAM. Unauthorized reproduction of this article is prohibited. 
has a unique solution $\left(\theta^{*}, \eta^{*}\right)$, where

$$
\Phi_{\theta}^{e}=\left[\Phi_{0}^{e} \theta, \Phi_{1}^{e} \theta, \ldots, \Phi_{m}^{e} \theta\right] .
$$

Let

$$
\begin{gathered}
\zeta^{e}(N)=\left[\zeta_{1}^{e}(N), \ldots, \zeta_{n(m+1)}^{e}(N)\right]^{T} \\
V_{m}^{e}=\mathbf{T}\left(q^{m},\left[v_{n}^{m}, \ldots, v_{1}^{m}\right]\right), \quad\left[r_{1}, \ldots, r_{m}\right]:=\left(\Re^{T}\right)^{-1} \\
\Xi^{e}(N)=\left(\begin{array}{cccc}
\zeta_{1}^{e}(N) & \zeta_{2}^{e}(N) & \ldots & \zeta_{n}^{e}(N) \\
\zeta_{n+1}^{e}(N) & \zeta_{n+2}^{e}(N) & \cdots & \zeta_{2 n}^{e}(N) \\
& & \vdots & \\
\zeta_{m n+1}^{e}(N) & \zeta_{m n+2}^{e}(N) & \cdots & \zeta_{n(m+1)}^{e}(N)
\end{array}\right)
\end{gathered}
$$

Then, we have the following identification algorithm:

(i) Estimation of $\theta$. The estimate of $\theta$ is taken as

$$
\theta^{e}(N)=\left(\Phi_{m}^{e}\right)^{-1}\left(\Xi^{e}(N)\right)^{T} r_{m}
$$

(ii) Estimation of $\eta$. Let $b_{j}^{e}(0)=0$, and let

$$
b_{j}^{e}(N)= \begin{cases}{\left[\zeta_{i_{j}^{e}(N)}^{e}, \zeta_{2 n+i_{j}^{e}(N)}^{e}, \ldots, \zeta_{2 m n+i_{j}^{e}(N)}^{e}\right] \frac{r_{i_{j}^{e}(N)}^{e}}{\tau_{j, i_{j}^{e}}^{e}(N)},} & \tau_{j, i_{j}^{e}}^{e}(N) \neq 0, \\ b_{j}^{e}(N-1), & \tau_{j, i_{j}^{e}}^{e}(N)=0,\end{cases}
$$

where

$$
i_{j}^{e}(N)=\min \left\{\operatorname{argmax}_{1 \leq i \leq n}\left|\tau_{j, i}^{e}(N)\right|\right\}, \quad j=0,1, \ldots, m-1,
$$

$r_{i_{j}^{e}(N)}$ is the $i_{j}^{e}(N)$ th column of $\left(\Re^{T}\right)^{-1}$, and $\tau_{j, i_{j}^{e}}^{e}(N)$ is the $i_{j}^{e}(N)$ th component of

$$
\tau_{j}^{e}(N)=V_{j}^{e} \theta(N)
$$

Then, the estimate of $\eta$ is taken as

$$
\eta^{e}(N)=\left[b_{0}^{e}(N), \ldots, b_{m-1}^{e}(N)\right]^{T} .
$$

TheORem 6.4. Suppose $u \in \mathcal{U}_{q}(m)$. Then, under Assumptions A1 and A2,

$$
\theta^{e}(N) \rightarrow \theta, \eta^{e}(N) \rightarrow \eta \quad \text { w.p.1 as } N \rightarrow \infty .
$$

7. Illustrative examples. In this section, we use several examples to illustrate convergence of the estimates given by the algorithms developed in this paper. In all examples, the noise is Gaussian with known mean and variance. In Example 7.1, convergence of the basic identification algorithm for a gain with quantized observations is demonstrated. Example 7.2 concerns identification of a Hammerstein system with a nonmonotonic nonlinearity. Example 7.3 illustrates that sometimes additional prior information on unknown system parameters can be utilized to simplify algorithms.

Example 7.1. Consider a gain system $y(k)=a+d(k)$. Here the actual value of the unknown $a$ is 5 . The disturbance is a sequence of i.i.d. Gaussian variables 


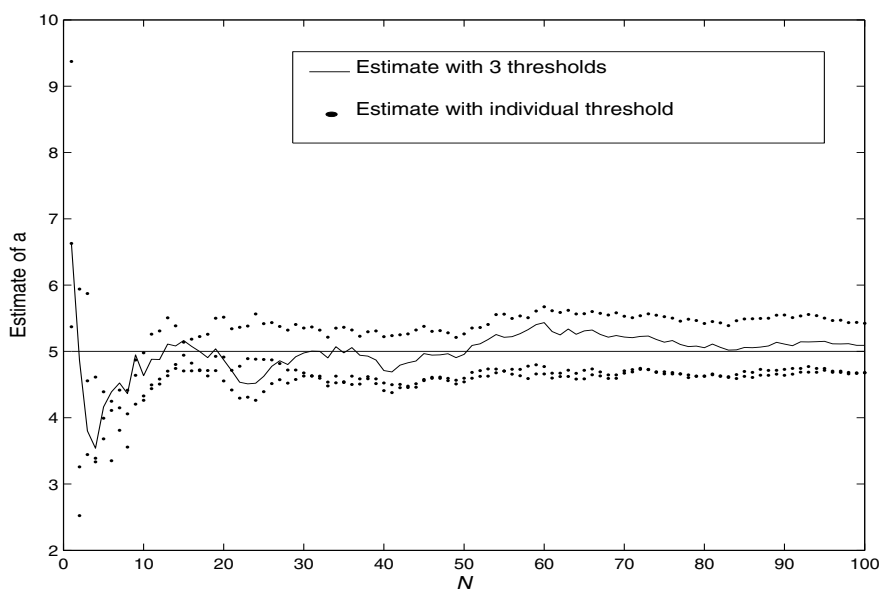

FIG. 2. Identification with quantized output observations.

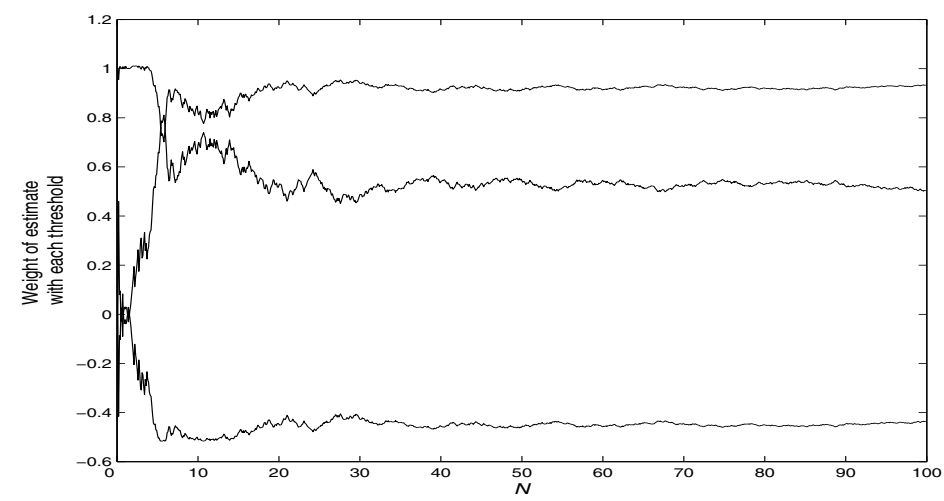

FIG. 3. Weights of estimates with each threshold.

with zero mean and standard deviation $\sigma=5$. The sensor has 3 switching thresholds $C_{1}=2, C_{2}=6$, and $C_{3}=10$. Then, the recursive algorithm in section 5 is used to generate quasi-convex combination estimates. For comparison, estimates derived by using each threshold individually (i.e., binary-valued sensors) are also calculated. Figure 2 compares quasi-convex combination estimates to those using each threshold. It is shown that the estimate with three thresholds converges faster than those with each threshold individually. The weights of the estimates of each threshold are shown in Figure 3, which illustrates that the weights are not always positive.

Example 7.2. Consider

$$
\left\{\begin{array}{l}
y(k)=a_{0} x(k)+a_{1} x(k-1)+d(k), \\
x(k)=b_{0}+b_{1} u(k)+b_{2} u^{2}(k)+u^{3}(k),
\end{array}\right.
$$

where the noise $\{d(k)\}$ is a sequence of i.i.d. Gaussian variables with $E d_{1}=0, \sigma_{d}^{2}=1$. The output is measured by a binary-valued sensor with threshold $C=13$. The linear subsystem has order $n=2$, and the nonlinear function has order $m=2$. The prior information on $a_{i}, i=0,1$, is that $a_{i} \in[0.5,5]$. Suppose the true values of unknown parameters are $\theta=\left[a_{0}, a_{1}\right]^{T}=[1.31,0.85]^{T}$ and $\eta=\left[b_{0}, b_{1}, b_{2}\right]^{T}=[4,1.4,-3]^{T}$. The 


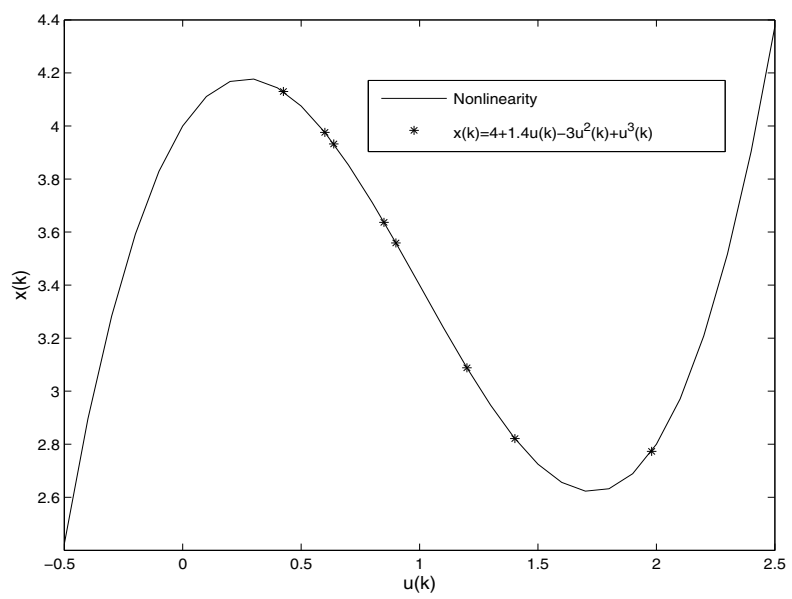

FIG. 4. Nonmonotonic nonlinearity.

nonlinearity is not monotone, which is illustrated in Figure 4. It is shown that not all values of $v, \rho_{1} v, \rho_{2} v, \rho_{3} v$ are situated in the same monotone interval of the nonlinearity.

The input is chosen to be $2 n(m+1)=12$-periodic with one-period values $\left(v, v, \rho_{1} v, \rho_{1} v, \rho_{2} v, \rho_{2} v\right)$, where $v=[1.2,0.85], \rho_{1}=0.5, \rho_{2}=1.65$, and $\rho_{3}=0.75$. Define the block variables $X(l), Y(l), \Phi_{j}(l), D(l)$, and $S(l)$ in the case of a 6-periodic input. Using (4.9), we can construct the algorithms from subsection 6.2 to identify $\theta$ and $\eta$.

The estimate errors of $\theta$ and $\eta$ are illustrated in Figure 5, where the errors are measured by the Euclidean norm. The parameter estimates of both the linear and nonlinear subsystems converge to their true values, despite that the nonlinearity is not monotonic.

Example 7.3. For some prior information, the algorithms in subsection 6.2 can be simplified. For example, the estimate algorithms of $\eta$ can be simplified when the prior information on $\theta$ is known to be positive and the periodic input $u$ is positive. Both the mean and the variance of disturbance are not zero in this example.

Consider

$$
\left\{\begin{array}{l}
y(k)=a_{0} x(k)+a_{1} x(k-1)+d(k), \\
x(k)=b_{0}+b_{1} u(k)+u^{2}(k),
\end{array}\right.
$$

where the noise $\{d(k)\}$ is a sequence of i.i.d. Gaussian variables with $E d_{1}=2, \sigma_{d}^{2}=4$. The output is measured by a binary-valued sensor with threshold $C=13$. The linear subsystem has order $n=2$, and the nonlinear function has order $m=2$. The prior information on $a_{i}, i=0,1$, is that $a_{i} \in[0.5,5]$. Suppose the true values of unknown parameters are $\theta=\left[a_{0}, a_{1}\right]^{T}=[1.17,0.95]^{T}$ and $\eta=\left[b_{0}, b_{1}\right]^{T}=[3,1.3]^{T}$.

The input is 12-periodic with one-period $\left(v, v, \rho_{1} v, \rho_{1} v, \rho_{2} v, \rho_{2} v\right)$, where $v=$ $[1.2,0.85], \rho_{1}=0.65$, and $\rho_{2}=1.25$. Define the block variables $X(l), Y(l), \Phi_{j}(l)$, $D(l)$, and $S(l)$ in the case of a 12-periodic input. Using (4.9), we can construct the algorithms in subsection 6.2 to identify $\theta$.

Considering the prior information on $\theta$, a more simplified algorithm than the one in subsection 6.2 can be constructed to identify $\eta$. Notice that $a_{i} \in[0.5,5], i=1,2$, 

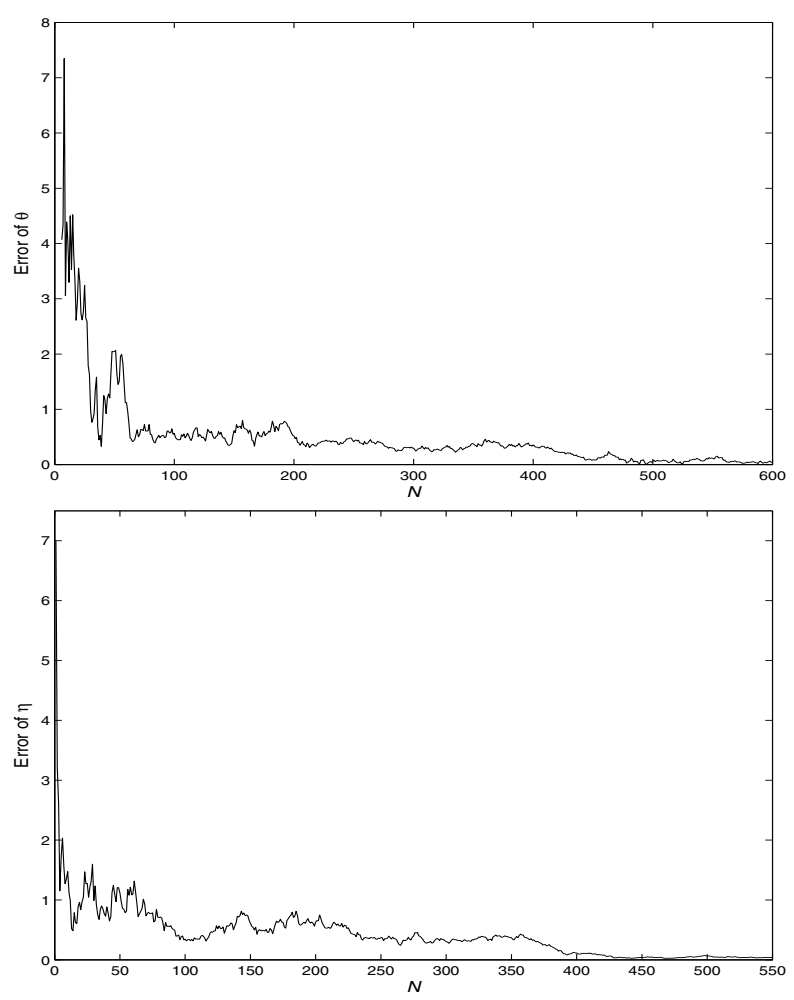

FIG. 5. Identification errors of $\theta$ and $\eta$ with nonmonotonic nonlinearity.

and $u$ is positive. Then, $\tau_{j, 1}$, the first component of $V_{j} \theta$, is

$$
\tau_{j, 1}=v_{2}^{2} a_{0}+v_{1}^{2} a_{1} \geq 0.5\left(v_{2}^{2}+v_{1}^{2}\right) \neq 0,
$$

where the last inequality is derived from the fact that $v$ is strongly 2 full rank. So, it is not necessary to calculate $i_{j}^{*}(N)$ in $(6.3)$, which aims to find the nonzero component of $\tau_{j}$. And $\eta$ can be estimated as

$$
\eta(N)= \begin{cases}\Lambda(N) \Re^{c}\left[\zeta_{1}(N), \zeta_{2 n+1}(N), \ldots, \zeta_{2 m n+1}(N)\right]^{T} & \text { if } \prod_{j=0}^{m-1} \tau_{j, 1}(N) \neq 0, \\ \eta(N-1) & \text { if } \prod_{j=0}^{m-1} \tau_{j, 1}(N)=0,\end{cases}
$$

where $\Lambda(N)=\operatorname{diag}^{-1}\left(\tau_{0,1}(N), \ldots, \tau_{m-1,1}(N)\right), \Re^{c}$ is an $m \times(m+1)$ matrix containing from 1st to $m-1$ st rows of $\Re^{-1}$, and $\tau_{j, 1}(N)$ is the 1st component of $\tau_{j}(N)=V_{j} \theta(N)$.

The estimation errors of $\theta$ and $\eta$ are shown in Figure 6, where the errors are measured by the Euclidean norm. Parameter estimates of both the linear and nonlinear subsystems converge to their true values.

8. Concluding remarks. In this paper, identification of Hammerstein systems with quantized output observations is studied. Unlike traditional approximate gradient methods or covariance analysis, we employ the methods of empirical measures and 

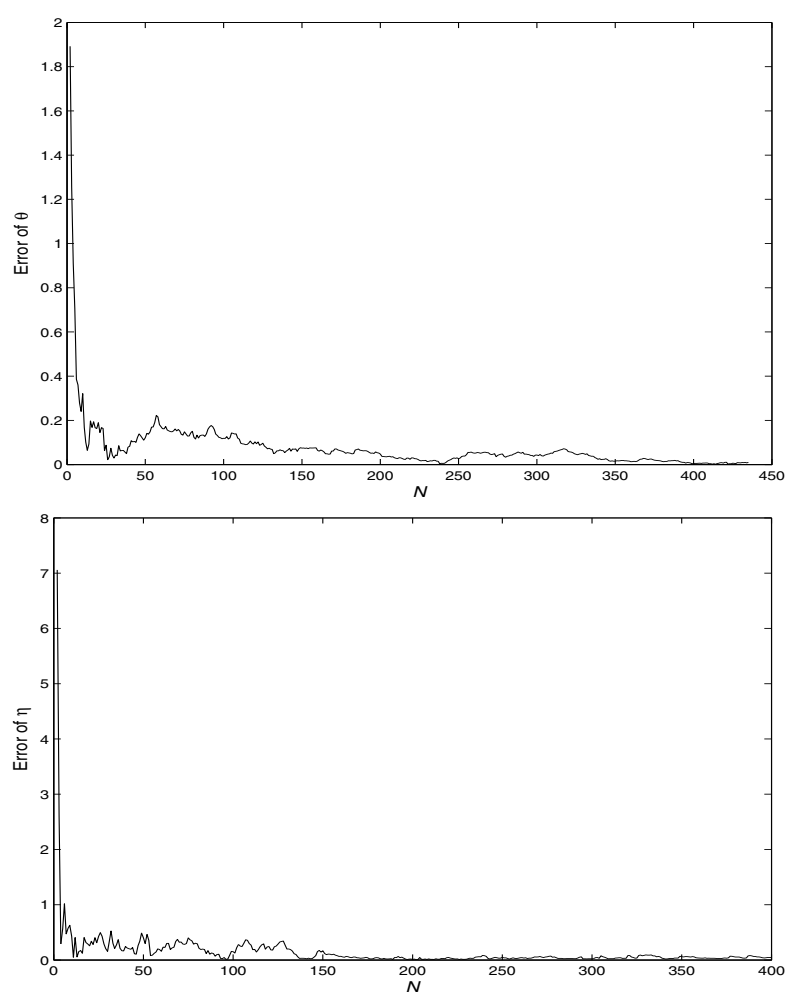

FIG. 6. Identification errors of $\theta$ and $\eta$.

parameter mappings. Under assumptions of known disturbance distribution functions and strongly scaled full rank inputs, identification algorithms, convergence properties, and estimation efficiency are derived.

In this paper, we assume that the nonlinearity is polynomial and that the order of the linear dynamics and nonlinear function are known. The issues of unmodeled dynamics (for the linear subsystem when the system order is higher than the model order) and model mismatch (for the nonlinear part when the nonlinear function does not belong to the model class) are not covered here, mainly due to page limitation. Interested readers are referred to [24, 25], where some related results on irreducible identification errors due to unmodeled dynamics and impact of model mismatch on identification errors, etc. can be found.

There are many potential extensions of the results in this paper. For example, when the sensor threshold value and/or the noise distribution functions are unknown, combined identification of systems, distribution functions, and sensor thresholds is of practical importance. Some related results can be found in [22]. Also, more general nonlinear functions may be considered.

\section{REFERENCES}

[1] E. W. BAI, An optimal two-stage identification algorithm for Hammerstein-Wiener nonlinear system, Automatica J. IFAC, 34 (1998), pp. 333-338.

[2] E. W. BAI, A blind approach to the Hammerstein-Wiener model identification, Automatica J. IFAC, 38 (2002), pp. 967-979. 
[3] P. Billingsley, Convergence of Probability Measures, John Wiley, New York, 1968.

[4] S. Billings, Identification of nonlinear systems - a survey, Proc. IEE-D, 127 (1980), pp. 272285.

[5] H.-F. CHEN, Recursive identification for Wiener model with discontinuous piece-wise linear function, IEEE Trans. Automat. Control, 51 (2006), pp. 390-400.

[6] Y. S. Chow, Probability Theory, Springer-Verlag, New York, 1978.

[7] X. L. Hu AND H. F. Chen, Strong consistence of recursive identification for Wiener systems, Automatica J. IFAC, 41 (2005), pp. 1905-1916.

[8] I. W. Hunter And M. J. KorenBerg, The identification of nonlinear biological systems: Wiener and Hammerstein cascade models, Biol. Cybernet., 55 (1986), pp. 135-144.

[9] A. Juditsky, H. Hualmarsson, A. Benveniste, B. Delyon, L. Ljung, J. Sjoberg, and Q. ZHANG, Nonlinear black box models in system identification: Mathematical foundations, Automatica J. IFAC, 31 (1995), pp. 1725-1750.

[10] M. J. Korenberg, Identification of biological cascades of linear and static nonlinear systems, in Proceedings of the 16th Midwest Symposium on Circuit Theory, Waterloo, Ontario, 18 (1973), pp. 1-9.

[11] M. J. Korenberg, Recent advances in the identification of nonlinear systems: Minimumvariance approximation by Hammerstein models, in Proceedings of the Annual International Conference of the IEEE Engineering in Medicine and Biology Society, Orlando, FL, Vol. 13, 1991, pp. 2258-2259.

[12] M. J. Korenberg And I. W. Hunter, Two methods for identifying Wiener cascades having noninvertible static nonlinearities, Ann. Biomed. Engrg., 27 (1998), pp. 793-804.

[13] H. J. Kushner And G. Yin, Stochastic Approximation and Recursive Algorithms and Applications, 2nd ed., Springer-Verlag, New York, 2003.

[14] S. L. LaCy And D. S. Bernstein, Identification of FIR Wiener systems with unknown, noninvertible polynomial nonlinearities, Internat. J. Control, 76 (2003), pp. 1500-1507.

[15] P. Lancaster and M. Tismenetsky, The Theory of Matrices, 2nd ed., Academic Press, New York, 1985.

[16] L. LJUnG, Convergence analysis of parametric identification methods, IEEE Trans. Automat. Control, 23 (1978), pp. 770-783.

[17] L. LuUng AND P. E. CAINes, Asymptotic normality of prediction error estimators for approximate system models, Stochastics, 3 (1979), pp. 29-46.

[18] K. NARENDRA AND P. Gallman, An iterative method for the identification of nonlinear systems using a Hammerstein model, IEEE Trans. Automat. Control, 11 (1966), pp. 546-550.

[19] B. Ninness And S. GiBSOn, Quantifying the accuracy of Hammerstein model estimation, Automatica J. IFAC, 38 (2002), pp. 2037-2051.

[20] R. J. Serfling, Approximation Theorems of Mathematical Statistics, John Wiley, New York, 1980.

[21] L. Y. WANG AND G. YIN, Asymptotically efficient parameter estimation using quantized output observations, Automatica J. IFAC, 43 (2007), pp. 1178-1191.

[22] L. Y. WANG, G. YIN, AND J. F. ZHANG, Joint identification of plant rational models and noise distribution functions using binary-valued observations, Automatica J. IFAC, 42 (2006), pp. 535-547.

[23] L. Y. Wang, G. Yin, J. F. Zhang, and Y. L. ZhaO, Space and time complexities and sensor threshold selection in quantized identification, Automatica J. IFAC, 44 (2008), pp. 30143024.

[24] L. Y. WAng, J. F. Zhang, AND G. Yin, System identification using binary sensors, IEEE Trans. Automat. Control, 48 (2003), pp. 1892-1907.

[25] G. G. YIN, S. KAN, AND L. Y. WANG, Identification error bounds and asymptotic distributions for systems with structural uncertainties, J. Syst. Sci. Complex., 19 (2006), pp. 22-35.

[26] Y. L. ZhaO, L. Y. WANG, G. G. Yin, AND J. F. Zhang, Identification of Wiener systems with binary-valued output observations, Automatica J. IFAC, 43 (2007), pp. 1752-1765.

Copyright $@$ by SIAM. Unauthorized reproduction of this article is prohibited. 